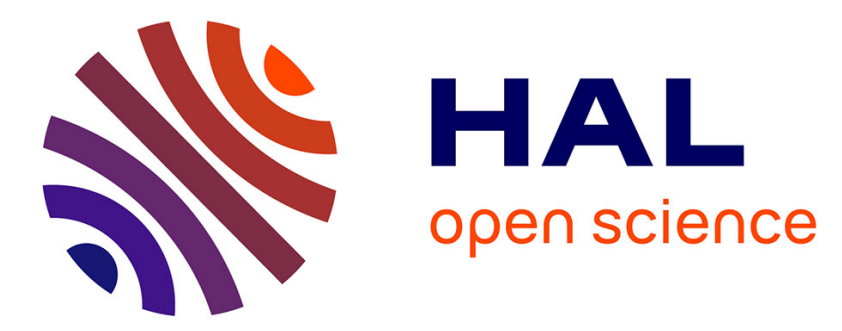

\title{
Accelerating multi-modal image registration using a supervoxel-based variational framework
}

L Lafitte, Cornel Zachiu, Linda Kerkmeijer, Mario Ries, Baudouin Denis de Senneville

\section{- To cite this version:}

L Lafitte, Cornel Zachiu, Linda Kerkmeijer, Mario Ries, Baudouin Denis de Senneville. Accelerating multi-modal image registration using a supervoxel-based variational framework. Physics in Medicine and Biology, 2018, 63 (23), pp.235009. hal-01962461

\section{HAL Id: hal-01962461 \\ https://hal.science/hal-01962461}

Submitted on 21 Dec 2018

HAL is a multi-disciplinary open access archive for the deposit and dissemination of scientific research documents, whether they are published or not. The documents may come from teaching and research institutions in France or abroad, or from public or private research centers.
L'archive ouverte pluridisciplinaire HAL, est destinée au dépôt et à la diffusion de documents scientifiques de niveau recherche, publiés ou non, émanant des établissements d'enseignement et de recherche français ou étrangers, des laboratoires publics ou privés. 


\title{
Accelerating multi-modal image registration using a supervoxel-based variational framework
}

\author{
L Lafitte ${ }^{1}$, C Zachiu ${ }^{3}$, L G W Kerkmeijer ${ }^{3}$, M Ries ${ }^{2}$, and B \\ Denis de Senneville ${ }^{1,3}$ \\ 1 "Institut de Mathématiques de Bordeaux", University of Bordeaux/CNRS UMR \\ 5251, 351 Cours de la Libération, 33405 Talence Cedex, France \\ ${ }^{2}$ Imaging Division, UMC Utrecht, Heidelberglaan 100, 3584 CX, Utrecht, \\ Netherlands \\ ${ }^{3}$ Department of Radiotherapy, UMC Utrecht, Heidelberglaan 100, 3508 GA, \\ Netherlands \\ E-mail: Luc.Lafitte@math.u-bordeaux.fr, C.Zachiu@umcutrecht.nl, \\ L.Kerkmeijer@umcutrecht.nl, M.Ries@umcutrecht.nl, \\ bdenisde@math.u-bordeaux.fr
}

May 2018

\begin{abstract}
For the successful completion of medical interventional procedures, several concepts, such as daily positioning compensation, dose accumulation or delineation propagation, rely on establishing a spatial coherence between planning images and images acquired at different time instants over the course of the therapy. To meet this need, image-based motion estimation and compensation relies on fast, automatic, accurate and precise registration algorithms. However, image registration quickly becomes a challenging and computationally intensive task, especially when multiple imaging modalities are involved.

In the current study, a novel framework is introduced to reduce the computational overhead of variational registration methods. The proposed framework selects representative voxels of the registration process, based on a supervoxel algorithm. Costly calculations are hereby restrained to a subset of voxels, leading to a less expensive spatial regularized interpolation process. The novel framework is tested in conjunction with the recently proposed EVolution multi-modal registration method. This results in an algorithm requiring a low number of input parameters, is easily parallelizable and provides an elastic voxel-wise deformation with a subvoxel accuracy.

The performance of the proposed accelerated registration method is evaluated on cross-contrast abdominal T1/T2 MR-scans undergoing a known deformation and annotated CT-images of the lung. We also analyze the ability of the method to capture slow physiological drifts during MR-guided high intensity focused ultrasound therapies and to perform multi-modal $\mathrm{CT} / \mathrm{MR}$ registration in the abdomen. Results have shown that computation time can be reduced by $75 \%$ on the same hardware with no negative impact on the accuracy.
\end{abstract}

Keywords: Multi-modal registration, Non-rigid registration, Supervoxel, Variational 
method

Submitted to: Phys. Med. Biol. 


\section{Introduction}

Over the course of interventional procedures such as external beam radiotherapy (EBRT) (Guckenberger et al. 2012) or high intensity focused ultrasound (HIFU) (Jolesz \& Hynynen 2002), the therapeutic work-flow is frequently hampered by organ motion. Daily positioning errors and physiological activities generate spatial inconsistencies between images acquired at different times, which are problematic for inter- and/or intra-fractional dose accumulation or delineation propagation (Rubeaux et al. 2013, Brock et al. 2017). A feasible solution for addressing such deformations is image registration, which is defined as the process of aligning two images acquired from different perspectives, with different sensors and/or different moments in time (Zitová \& Flusser 2003). However, EBRT and HIFU workflows frequently involve multiple imaging devices and/or contrasts thus, a multi-modal image registration tool is mandatory (Maes et al. 1997, Viola \& Wells III 1997, Pluim et al. 2003), which in turn can become a complex and computationally intensive task. This may be problematic, since compensating for the effects of motion during such therapies have to imply minimal temporal latencies in order to ensure a smooth workflow. Another difficulty arises in the abdomen, where a large image field-of-view (FOV) is needed to delineate and simulate the therapeutic dose deposition in both the tumor and the organs-at-risk. A local voxelwise deformation is thus needed to cope with elastic organ deformations, such as the liver and kidneys, as well as individual spatial displacements of surrounding tissues/anatomical structures.

A particular variety of registration algorithms are the so-called variational methods which have attracted a considerable attention over recent years in signal/image processing literature (Haddad \& Meyer 2004). In particular, such methods are widely used for image registration (Weickert et al. 2003) due to both their fast numerical schemes and the reduced number of required input parameters. The latter advantage renders these methods an increased ease-of-use in a clinical setting on a case-by-case basis. Considering $J$ to be an image that we aim to register to a reference position characterized by another image $I$ and $T$ to be a voxelwise deformation, variational methods involve:

(i) A data fidelity term (referred to as $D(T)$ in the scope of this study), which is a measure of the similarity between $I$ and $J$, the latter being registered using the spatial transformation $T$. $D(T)$, which is computed individually at each spatial location $\vec{r}$, is a strictly positive number and has to be designed to increase with the misalignment between $I$ and $J$.

(ii) A regularization term (referred to as $R(T)$ in the scope of this study), which constrains the estimated deformation. This renders the optimization problem overdetermined and therefore solvable using variational approaches.

The voxelwise transformation $T$ is found through the general formulation in form of an inverse problem which can be mathematically expressed as follows: 


$$
E(T)=\int_{\Omega} D(T)+\alpha R(T) \mathrm{d} \vec{r}
$$

$E(T)$ being the functional to minimize, $\Omega$ the image coordinates domain, $\vec{r}$ the spatial location and $\alpha$ an input parameter designed to weight the two contributions $D(T)$ and $R(T)$. The sought for transformation is found through an optimization process in which $T, D(T)$ and $R(T)$ are iteratively updated (Weickert et al. 2003).

Previous studies propose several variational methods dedicated to the estimation of dense and elastic deformations between images acquired with different image modalities and/or contrasts. A considerable part of these studies rely on the optimization of a data fidelity term defined by the mutual information (MI) between the registered images (Maes et al. 1997, Viola \& Wells III 1997, Studholme et al. 1999, Pluim et al. 2003, Rohlfing et al. 2003). In its original form, such an approach hypothesizes that there exists an invariant relationship between the image intensities of the same anatomical structures/tissues in the two images, over the entire image domain. In the absence of severe local intensity inhomogeneities this has been proven to be a reliable approach, with more recent studies aiming to localize the MI calculation in order to improve its robustness against such phenomena (Studholme et al. 2006, Klein et al. 2008, Loeckx et al. 2010, Rivaz et al. 2014). A different more recent multimodal registration approach has been proposed by Heinrich et al, based on so-called modality independent neighborhood descriptors (MIND) (Heinrich et al. 2012, Degen \& Heinrich 2016). The method transforms the input images into a vectorial representation such that a descriptor is associated to each voxel, characterizing its relationship to the neighboring voxels regardless of potential local contrast variations. A data fidelity term suitable for multi-modal image registration, called normalized gradient fields (NGF), was also proposed by Haber \& Modersitzki (Haber \& Modersitzki 2006) and adopted by several independent studies (e.g. (König et al. 2014, Hodneland et al. 2014)). The approach relies on the hypothesis that structural information within the images can be defined by intensity changes. In essence, this implies that the spatial correspondence between multi-modal images is found by searching for a transformation which aligns similar structures within the images. A similar hypothesis that the principal contrast patterns of soft-tissue structures are generally preserved across different image modalities/contrasts is also used by the EVolution algorithm proposed by our group (Denis de Senneville et al. 2016). Common advantages of both MIND, NGF and EVolution lie on their fast numerical schemes, low number of input parameters and their capability of estimating dense and elastic deformations. In addition, EVolution also offers specific tracking capability in scenarios for which structures in the image to register do not have counterparts in the reference image. The EVolution algorithm was recently tested with various application scenarios such as T1/T2-MR (Denis de Senneville et al. 2016) and CT/CBCT registration (Zachiu et al. 2017b), as well as real-time target US-based tracking dedicated to HIFU therapies (Zachiu et al. 2017c).

While employing various regularization terms such as smoothness, curvature or 
incompressibility, the computational demand of the above-mentioned multi-modal registration algorithms remains predominantly determined by the calculation of the data fidelity term. In the context of a clinical workflow, the computation time is a critical point and several minutes at least are typically required for the registration of $3 \mathrm{D}$ images of dimension $128 \times 128 \times 128$ using above mentioned methods.A simple manner to accelerate the registration process consists in running the algorithms on a down-sampled version of the input images (Glitzner et al. 2015). This comes, however at a cost in precision and accuracy of the motion estimates due to a loss of image features or occurrence of artifacts during the down-sampling process. In this paper, we propose to reduce the amount of voxels on which the data fidelity term is calculated. A sparse, and compact data fidelity term (noted $D_{s}(T)$ ) is first obtained. Although $D_{s}(T)$ offers the advantage to be low time consuming, it cannot be directly used in Eq. (1) because it is not defined at every voxel. A regularized interpolation procedure is thus employed to convert $D_{s}(T)$ into a voxelwise data fidelity term $D(T)$, assuming that $D(T)$ is spatially smooth. The variational functional of Eq. (1) is then optimized using the regularized data fidelity term $D(T)$. For this purpose, the two following image/signal processing strategies are employed:

(i) Supervoxel clustering: These algorithms aim at reducing the redundancies present in an image (Achanta et al. 2012). Generally, the objective is to increase the efficiency, in terms of both accuracy and/or reduced computational costs, of a specific subsequent processing task, such as object recognition, image segmentation (Veksler et al. 2010) or optical flow image registration (Gkamas \& Nikou 2011) (Donné et al. 2015). In our study, a supervoxel clustering method is employed to calculate a sparse and compact set of voxels of interest on which the data fidelity term is calculated.

(ii) Regularized interpolation from sparse inputs: We interpolated the abovementioned sparse data fidelity term over the complete image FOV by means of a standard Tikhonov regularization (Ramani et al. 2010).

Our contribution is four-fold:

(i) A method is proposed for the calculation of a sparse compact data fidelity term at each iteration of the registration process. A regularized interpolation technique is then used to assess the data fidelity term over the complete FOV with a low computational overhead. The resulting interpolated data fidelity term $D(T)$ is then used in Eq. (1) in order to estimate the deformation $T$. Note that the proposed accelerated framework is expected to generalize to other variational formulations of the registration problem.

(ii) The performance of the proposed accelerated registration method is compared, in terms of both precision and computational overhead, to the original version of the EVolution algorithm. For this purpose, our experimental setup included crosscontrast abdominal T1/T2 MR scans with an imposed deformation and annotated 
thoracic CT-images acquired on esophagus cancer patients. The proposed approach is also employed for the estimation of slow physiological drifts occurring over the duration of an MR-guided HIFU therapy and for the registration of abdominal $\mathrm{CT} / \mathrm{MR}$ volumes.

(iii) The improvement of the proposed framework in terms of accuracy/computation time is demonstrated as compared to motion estimates from down-sampled images.

(iv) The benefit of using multi-CPU and GPU (graphics processing unit) architectures is evaluated for real-time applications.

\section{Materials and Methods}

\subsection{Proposed accelerated variational framework}

The proposed processing sequence (detailed in Fig. 1) iteratively updates the displacement estimates $T$ via the following steps:

(i) A supervoxel-based image clustering is first calculated for both the reference $I$ and the image to register $J$, the latter being registered using the ongoing deformation estimate $T$ (for the remainder of the paper the registered image $J$ is referred to as $J(T))$.

(ii) Based on the two sets of supervoxels (with each supervoxel being a set of voxels with similar contrast properties), a sparse and compact collection of voxels of interest is calculated.

(iii) A data fidelity term, which is designed for multi-modal image registration, is calculated on the sparse voxel collection.

(iv) The obtained sparse data fidelity term $\left(D_{s}(T)\right)$ is interpolated over the complete image FOV using a standard Tikhonov regularization (Ramani et al. 2010).

(v) The obtained interpolated data fidelity term $(D(T))$ is used in Eq. (1) to update the estimation of the voxelwise deformation $T$.
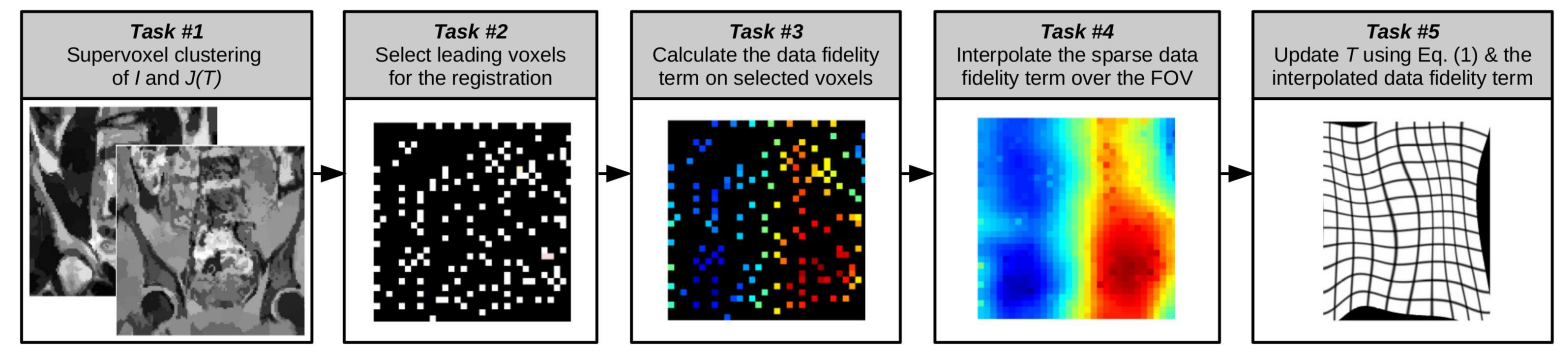

Figure 1: Proposed accelerated variational framework designed to iteratively update the geometric deformation $T$. 
2.1.1. Clustering input images (Task \#1) First, $I$ and $J(T)$ are independently clustered into supervoxels (i.e. a group of connected voxels with similar gray levels). For this purpose, we used the SLIC (Simple Linear Iterative Clustering) algorithm (Achanta et al. 2012) which aims at dividing images into non-overlapping supervoxels with a low computational overhead. In our study, the use of supervoxel segmentation has two advantages: first, voxels present in supervoxel borders are likely to match organ edges present in the image (while voxels located within an identical supervoxel are selected to have similar gray levels, voxels with largest gray level variations in the vicinity are likely to be located astride supervoxels). Second, supervoxels fully recover the complete image field-of-view which is an essential property for the subsequent regularized interpolation process (Task \#4).

Let $N$ be the desired number of supervoxels, and $\gamma$ the voxel decimation ratio (i.e the ratio between the number of supervoxels and the amount of voxels in the original image: $\left.\gamma=\frac{N}{\operatorname{Card}(\Omega)}, \gamma \in[0,1]\right)$. We used $\gamma$ as an input parameter of the algorithm instead of $N$ in order to mitigate the impact of the working image dimension onto the supervoxels clustering results.

2.1.2. Determination of a sparse subset of voxels (Task \#2) Here, a sparse and compact voxels distribution over the entire image FOV is constituted. For a better comprehension of this step it should be noted that the resulting data fidelity term employed later in the framework (Task \#3, section 2.1.3) aims at maximizing edge alignment between the images being registered. Leading voxels for the registration are therefore preferably located on corners/contours present in both $I$ and $J(T)$. As a consequence, our algorithm grants the highest priority to voxels located astride largest number of supervoxels. The rank of a voxel was thereby given by the number of separate supervoxels in its 6-connected neighborhood (i.e left/right/up/down/front/back adjoining voxels). For each image being registered, voxels having a maximal rank of 6 were first added in a list. Lower ranks were then enumerated in descending order: associated voxels were added in the list, until the size of the latter exceeds chosen number of supervoxels $N$. The sparse subset of voxels was constituted by the two obtained lists (each list arising from $I$ and $J(T)$, respectively).

2.1.3. Calculation of a sparse data fidelity term (Task \#3) The data fidelity term is then evaluated on the obtained sparse voxel collection obtained by the previous step and denote by $D_{s}(T)$ the obtained sparse data fidelity term. For this purpose, we used the multi-modal data fidelity term proposed in (Denis de Senneville et al. 2016), which is expressed as follows:

$$
D_{s}(T)=e^{-C(T)}
$$

with: 


$$
C(T)=\frac{\int_{\Gamma}\left|\vec{\nabla}_{I}(T(\vec{r})) \cdot \vec{\nabla}_{J}(\vec{r})\right| \mathrm{d} \vec{r}}{\int_{\Gamma}\left\|\vec{\nabla}_{I}(T(\vec{r}))\right\|_{2}\left\|\vec{\nabla}_{J}(\vec{r})\right\|_{2} \mathrm{~d} \vec{r}}
$$

where $\Gamma$ is a cubic local neighborhood around the voxel being processed. More intuitively, Eq. (3) can be rewritten in 2D as follows:

$$
C(T)=\frac{\int_{\Gamma} w_{T}(\vec{r})\left|\cos \left(\Delta \theta_{T}(\vec{r})\right)\right| \mathrm{d} \vec{r}}{\int_{\Gamma} w_{T}(\vec{r}) \mathrm{d} \vec{r}}
$$

where $w_{T}(\vec{r})$ and $\Delta \theta_{T}(\vec{r})$ are calculated from the magnitude $M$ and the orientation $\theta$ of the image gradient at location $\vec{r}$.

Practically, the term $\left|\cos \left(\Delta \theta_{T}(\vec{r})\right)\right|$ in Eq. (4) quantifies the angle between the gradient orientations and favors parallel and anti-parallel gradients (and therefore, implicitly, similar local contrast patterns). On its side, $w_{T}(\vec{r})$ favors the alignment of edges/gradients that are present in both images. Due to the use of a decreasing exponential function in Eq. $(2), D_{s}(T)$ increases as long as the alignment of $I$ and $J(T)$ improves, as it is effectively required for the minimization of the functional in Eq. (1).

2.1.4. Regularized interpolation of the sparse data fidelity term (Task \#4) The voxelwise interpolated data fidelity term $D(T)$ can be obtained by interleaving in the registration process a second variational scheme, which aims at minimizing the following functional:

$$
F(D(T))=\int_{\Omega}\left|\xi D(T)-D_{s}(T)\right|+\frac{\mu}{2}\|\vec{\nabla} D(T)\|_{2}^{2} \mathrm{~d} \vec{r}
$$

where $\xi$ is the sparse sampling operator $\left(\xi=1\right.$ for non-zero voxels in $D_{s}(T), \xi=0$ otherwise).

$F(D(T))$ involves two contributions: a fidelity term on $D_{s}(T)$ (first term of the addition) and a regularization term on $D(T)$ (second term of the addition). $\mu$ is a regularization parameter designed to link the two contributions. The reader is referred to Appendix A for a complete description of the fast numerical scheme proposed for the minimization of the functional $F$ in Eq. (5) and an automatic determination of $\mu$.

2.1.5. Minimization of the functional designed for image registration (Task \#5) At this point, we have a data fidelity value $D(T)$ at each voxel present in the image FOV. However, we recall that the optimization of the data term alone is generally an ill-posed problem with an infinity of solutions. This drawback can be addressed for example by adding the following regularization term $R(T)$ to the functional in Eq. (1), as proposed in (Horn \& Schunck 1981): 


$$
R(T)=\frac{1}{2}\|\vec{\nabla} T(\vec{r})\|_{2}^{2}
$$

The functional $E(T)$ in Eq. (1) can then be optimized using an approach similar to the one proposed in (Denis de Senneville et al. 2016): the minimizer of $E(T)$ was found by solving the associated Euler-Lagrange equations. However, the differential nature of involved terms hampers the estimation of large displacements. In order to overcome this limitation and to reduce the non-linearity of the functional, a coarse-to-fine strategy is employed which iterates the algorithm from a down-sampled version of the images (sampling along minimal dimension $\geq 16$ voxels) step-by-step to the original resolution. Practically, motion estimates at each step are used as a starting point for the next higher resolution level. At each resolution level, the convergence of the algorithm was considered attained once the average difference between the displacements estimated at the current and the previous iteration is $\leq 10^{-3}$ voxels. The interested reader is referred to (Denis de Senneville et al. 2016) for additional information about the numerical resolution of Eq. (1).

\subsection{Experimental setup}

The proposed algorithm was evaluated in four complementary studies:

(i) Study \#1: A spatial transformation was analytically defined and subsequently applied on a T1-weighted MR image from a pair of simultaneously acquired T1/T2weighted images. The original T2 and the deformed T1 image were then employed as the reference $(I)$ and the image to register $(J)$, respectively. The imposed voxelwise deformation is used as a gold standard for the assessment of the registration.

(ii) Study \#2: Manually positioned landmarks in CT-images were used as reference for the assessment of the registration. In this experiment, only a subset of the spatial deformation is known (i.e the landmark locations). This experiment evaluates the capability of the investigated registration algorithm to estimate realistic thorax deformations.

(iii) Study \#3: The benefit of the algorithm in a clinical context was initially investigated by employing it for the estimation slow physiological drifts which frequently occur during lengthy MRg-HIFU therapies. This implied the registration of 3D T1 to T2-weighted images acquired in the scope of a motion compensation protocol, specifically dedicated to such interventions (described in detail within (Zachiu, Denis de Senneville, Ries \& Moonen 2015) and (Zachiu et al. 2017a)).

(iv) Study \#4: Since CT to MR image registration is a frequent requirement within EBRT workflows, the proposed registration algorithm was also employed and evaluated for the registration of five CT/T2-MR image pairs acquired on renal-cell carcinoma (RCC) patients. In this particular case, the validation of the algorithm was based on manual delineations made available by an experienced staff member. 
The upcoming sections provide details on these four studies, including the quality assessment criteria used for their evaluation (chosen in accordance with the gold standard available in each study) and the employed hardware platform.

2.2.1. Investigated registration algorithms The performance in terms of both computational overhead and accuracy of motion estimates is evaluated for the original and the proposed supervoxel-based EVolution algorithm (referred to as "EVo" and "SVEVo", respectively).

We also recall that it is common to accelerate the registration process by simply running the algorithm on down-sampled versions of the two input images (Glitzner et al. 2015, Zachiu et al. 2017c). The obtained deformation field is then upsampled at the original image resolution and the magnitude of each displacement vector is adjusted accordingly. In the scope of this study, the performance of EVo applied on a 2-fold down-sampled image is also included for comparison (and referred to as "DS-EVo" for the remainder of the manuscript).

EVo, SV-EVo and DS-EVo require as input two parameters: $\alpha$ (see Eq. (1)) and $\Gamma$ (see Eq. (4)). To clarify the benefits of the proposed SV-EVo on the overall results, identical values for $\alpha$ and $\Gamma$ were chosen for the three algorithms. In this paper, we selected $\alpha=0.5$ and $\Gamma=11 \times 11 \times 11$, as suggested in (Denis de Senneville et al. 2016).

\subsubsection{Description of selected data-sets}

Study \#1: Cross-constrast T1/T2 MR-scans undergoing a known deformation A pair of 3D T1/T2-weighted images were simultaneously acquired on the lower abdomen of a volunteer. The T1-weighted sequence employed the following parameters: $\mathrm{TE}=2 \mathrm{~ms}$, $\mathrm{TR}=4.3 \mathrm{~ms}$, image matrix $192 \times 192 \times 100,10^{\circ}$ flip angle, with an isotropic voxel size of $2 \times 2 \times 2 \mathrm{~mm}^{3}$. For the T2-weighted acquisition, the employed parameters were as follows: $\mathrm{TE}=130 \mathrm{~ms}, \mathrm{TR}=1000 \mathrm{~ms}$, reconstructed image size $512 \times 512 \times 133,90^{\circ}$ flip angle, with a voxel size of $0.5 \times 0.5 \times 1.5 \mathrm{~mm}^{3}$.

The pair of $3 \mathrm{D} \mathrm{T} 1 / \mathrm{T} 2$-weighted $\mathrm{MR}$ images were first resampled to a common $128 \times 128 \times 100$ image matrix (an isotropic voxel size of $2 \times 2 \times 2 \mathrm{~mm}^{3}$ was set) using a tricubic interpolation. A spatial deformation was subsequently simulated by a Free Form Deformation (FFD) model such that its displaced grid points are produced by perturbing a uniform grid of points. The grid size was $40 \times 40 \times 40$ and a random perturbation was applied on each grid point using a uniform distribution on $[-20,20]$ interval. Finally, the image to register $J$ was generated by applying the obtained FFD transform on the T1-weighted image.

Study \#2: Annotated thorax inhale and exhale CT-scans A set of five publicly available CT-scan pairs of almost similar image dimensions (in-plane dimension $256 \times 256$ voxels, slice number varying from 94 to 112) was used in the scope of this particular experiment. The data is part of the freely provided DIR-Lab database at the University of Texas 
(Castillo et al. 2009, Castillo et al. 2010a, Castillo et al. 2010b). Each scan pair was acquired on the thorax of patients treated for esophagian cancer, between inhale and exhale phase of the breathing cycle. For each CT-scan, 300 anatomical landmarks, which typically included vessel and bronchial bifurcations, have been annotated by three experts (inter-observer errors below $1 \mathrm{~mm}$ ), as reported in (Castillo et al. 2009).

Study \#3: Correction of slow physiological drifts during MR-guided clinical interventions Over the duration of lengthy MRg-HIFU therapies, abdominal organs may undergo long-term physiological drifts due to several factors such as muscle relaxation and/or digestive and metabolic activity. Such displacements were demonstrated to become significant over time intervals of several minutes and, if not taken into consideration, may impair attaining the therapeutic endpoint (Zachiu, Denis de Senneville, Ries \& Moonen 2015, Zachiu et al. 2017a).

In order to capture the aforementioned slow physiological drifts, 3D MR-images were periodically acquired on the abdomen four healthy volunteers. For each volunteer, two pairs of $3 \mathrm{D} \mathrm{T} 1 / \mathrm{T} 2$-weighted images, were acquired $\simeq 60 \mathrm{~min}$ apart and selected for the role of reference image and respectively image to register. During those $60 \mathrm{~min}$, each volonteer underwent the acquisition protocol described in (Zachiu et al. 2017a). The T1-weighted sequence employed the following parameters: $\mathrm{TE}=2 \mathrm{~ms}, \mathrm{TR}=4.3 \mathrm{~ms}$, image matrix $192 \times 192 \times 75$, with an isotropic voxel size of $2 \times 2 \times 2 \mathrm{~mm}^{3}, 10^{\circ}$ flip angle, resulting in an acquisition time of 60-90s, depending on the volunteer's breathing cycle reproducibility. For the T2-weighted acquisition, the employed parameters were as follows: spin-echo sequence, $\mathrm{TE}=130 \mathrm{~ms}, \mathrm{TR}=1442 \mathrm{~ms}, 90^{\circ}$ flip angle, image matrix $=512 \times 512 \times 133$, voxel size $=0.48 \times 0.48 \times 1.5 \mathrm{~mm}^{3}$, readout bandwidth $=890 \mathrm{~Hz}$.

The two pairs of 3D T1/T2-weighted images were first resampled to a common $192 \times 192 \times 75$ image matrix (an isotropic voxel size of $2 \times 2 \times 2 \mathrm{~mm}^{3}$ was set) using a tricubic interpolation. Subsequently, a gold standard motion field was estimated using a common contrast for both the reference and the image to register (the T1-weighted images were employed) using the following mono-modal optical flow (OF) functional:

$$
E_{O F}(T)=\int_{\Omega}\left|I_{x} u+I_{y} v+I_{z} w+I_{t}\right|+\alpha\left(\|\vec{\nabla} u\|_{2}^{2}+\|\vec{\nabla} v\|_{2}^{2}+\|\vec{\nabla} w\|_{2}^{2}\right) \mathrm{d} \vec{r}
$$

where $I_{x, y, z, t}$ are the spatio-temporal partial derivatives of the image voxel intensity. A complete description of the associated non-rigid registration framework can be found in (Zachiu, Papadakis, Ries, Moonen \& Denis de Senneville 2015). The obtained OFmotion field was taken as a reference for the evaluation of the proposed EVolution method applied on cross-contrast images (the T1- and respectively the T2-weighted images were employed for the reference and the image to register). The 3D slow physiological drifts were quantified separately for the liver and kidneys of each volunteer. In order to limit the analysis to these organs of interest, an active contour-based 
segmentation procedure was employed, which was subsequently manually refined by an experienced staff member.

Study \#4: Paired abdominal CT/MR-scans SV-EVo and EVo were subsequently employed for aligning five pairs of 3D CT/MR images acquired on the abdomen of renal cell carcinoma (RCC) patients. Note that this image data has been collected within the context of an ethical review board approved clinical study $\ddagger$. The MR images were acquired on a Philips Ingenia 1.5 T scanner using a T2-weighted turbo spin echo sequence employing the following parameters: $T R=523 \mathrm{~ms}, T E=80 \mathrm{~ms}$, flip angle $=$ $90^{\circ}$, image size $=288 \times 288 \times 53$, voxel size $=1.4 \times 1.4 \times 3 \mathrm{~mm}^{3}$, bandwidth readout $=478 \mathrm{~Hz}$. CT images were acquired on a Philips Brilliance Big Bore scanner and had a matrix and a voxel size of $512 \times 512 \times 123$ and $1.1 \times 1.1 \times 3 \mathrm{~mm}^{3}$. In order to ensure spatial consistency, all images were mapped prior to registration onto common lattice of size $256 \times 256 \times 128$ with an isotropic voxel volume of $2 \times 2 \times 2 \mathrm{~mm}^{3}$.

2.2.3. Quality assessment criteria for the estimated deformations The performance of the registration methods was evaluated against several criteria, chosen in accordance with the gold standard available for each of the above-mentioned studies:

Concerning studies \#1 and \#3, the gold standard is a set of displacement fields. The quality of the estimated motion was thus assessed using the voxel-wise error in flow endpoint (FEP) computed as follows (Baker et al. 2011):

$$
\mathrm{FEP}=\sqrt{\left(u-u_{r e f}\right)^{2}+\left(v-v_{r e f}\right)^{2}+\left(w-w_{r e f}\right)^{2}}
$$

$T=(u, v, w)$ being the estimated $3 \mathrm{D}$ displacement and $\left(u_{r e f}, v_{r e f}, w_{r e f}\right)$ the simulated deformation field (see section 2.2.2).

Concerning study \#2, the gold standard is a set of coordinates providing the location of manually positioned anatomical landmarks. By definition, the FEP relates two motion vector fields to one-another, reason for which, in this case, it was deemed to be a less suitable metric than the target registration error (TRE). The performance was thus here assessed using the TRE of the anatomical landmarks as follows (Maurer et al. 1997):

$$
\mathrm{TRE}=\sqrt{\left(x_{I}+u\left(\vec{r}_{I}\right)-x_{J}\right)^{2}+\left(y_{I}+v\left(\vec{r}_{I}\right)-y_{J}\right)^{2}+\left(z_{I}+w\left(\vec{r}_{I}\right)-z_{J}\right)^{2}}
$$

where $\vec{r}_{I}=\left(x_{I}, y_{I}, z_{I}\right)$ and $\vec{r}_{J}=\left(x_{J}, y_{J}, z_{J}\right)$ denote the anatomical landmark coordinates in $I$ and $J$, respectively.

Concerning the study \#4, the gold standard is a set of manually drawn contours. The Dice Similarity Coefficient (DSC) (Dice 1945) was thus used to determine the contour overlap for the body and kidneys, before and after registration. Such an

$\ddagger$ Ethical approval for the acquisition, usage and publication of the data was provided by the Ethics Board of the UMC Utrecht, Utrecht, The Netherlands. 
analysis showcases the capability of the investigated registration algorithms for organ contour alignment, which is representative for their performance in high-contrast areas. Mathematically, the DSC is defined as follows:

$$
D S C(A, B)=\frac{2|A \cap B|}{|A|+|B|}
$$

where $A$ and $B$ are the two contours whose overlap is determined and $|\cdot|$ corresponds to the number of voxels within a contour.

2.2.4. Hardware and implementation Our test platform was an Intel $3.4 \mathrm{GHz}$ i7-4770 workstation (8 cores) with 16 GB of RAM. The GPU was a NVidia GeForce GTX 770 with 2 GB of dynamic random-access memory (NVIDIA, Santa Clara, CA, USA). Both CPU and GPU implementations of the proposed EVolution algorithm were realized and tested. The CPU implementation was performed in $\mathrm{C}++$ and parallelized through multi-threading. The GPU implementation was realized using the compute unified device architecture (CUDA) (NVIDIA 2008).

\section{Results}

\subsection{Cross-contrast T1/T2 MR-scans undergoing a known deformation (Study \#1)}

Fig. 2 shows the interpolated data fidelity term obtained using the T1/T2 MR-scans detailed in section 2.2.2 for various voxel decimation factors $\gamma$. The supervoxel clustering of the two input images $I$ and $J$ (Task \#1) are displayed in Fig. 2a-d and 2e-h, respectively. For visualization, the contrast of each image was enhanced using a contrastlimited adaptive histogram equalization (CLAHE) (Zuiderveld 1994), and grey color of each voxel in the displayed images was set to the mean gray color of the supervoxel region. That way, each cluster can be visually distinguished. It can be observed that the employed supervoxel algorithm refines correctly the clusters in highly detailed anatomical regions (see $2 \mathrm{~d}$ and $2 \mathrm{~h}$ ), and that the image content is visibly preserved. The interpolated data fidelity maps $D(T)$, based on the values computed on the sparse subset of voxels (Task \#2 and \#3), are displayed in Fig. 2i-l. Several differences in the image content in $D(T)$ are observable for $0.01 \% \leq \gamma \leq 0.2 \%$ (mainly high frequencies).

A visualization of a registration result is provided in Fig. 3 (a voxel decimation factor $\gamma=1 \%$ was employed). The undeformed T1-weighted image is displayed along each image orientation in the first column. The simulated transformation (see section 2.2.2) is used to deform a rectilinear regular grid (only the in-plane deformation along each image orientation can be seen). The averaged FEP decreased from $6.15 \mathrm{~mm}$ before registration (third column) to $2.25 \mathrm{~mm}$ using the EVo (fourth column) and $2.24 \mathrm{~mm}$ using the proposed SV-EVo algorithm (fifth column).

Fig. 4 illustrates the performance of each tested algorithm in terms of accuracy of motion estimates (4a) and computation time (4b). A log-scale is employed for the $\mathrm{X}$-axis 


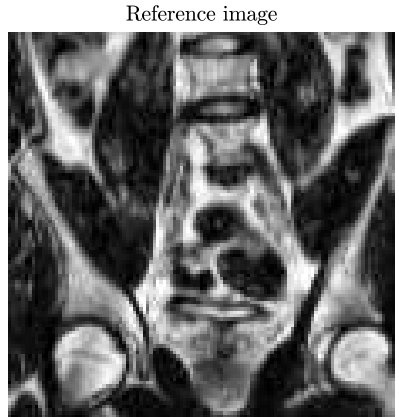

(a)

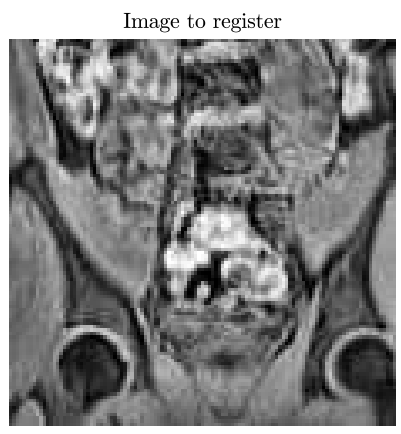

(e)

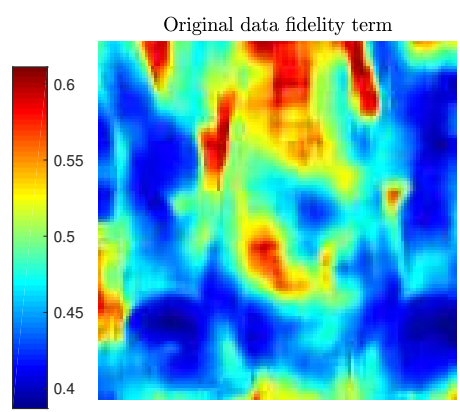

(i)

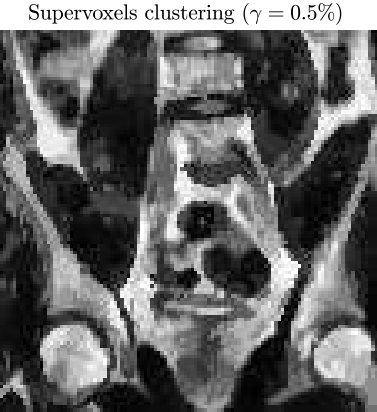

(b)

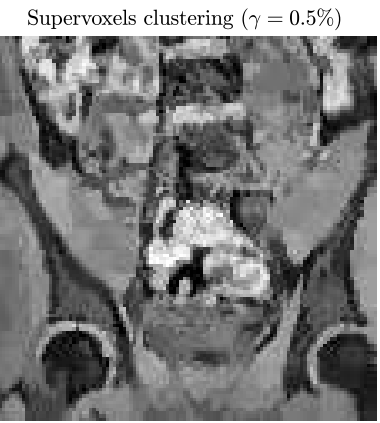

(f)

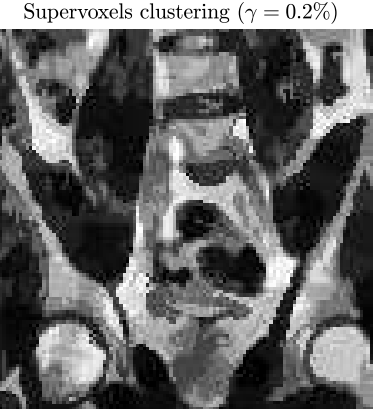

(c)

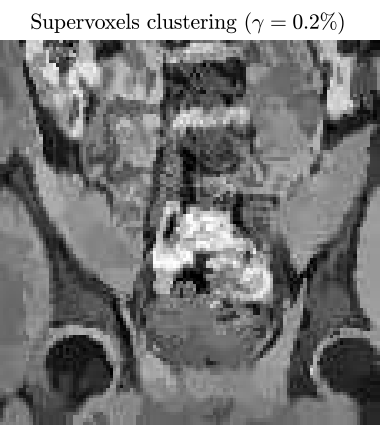

(g)

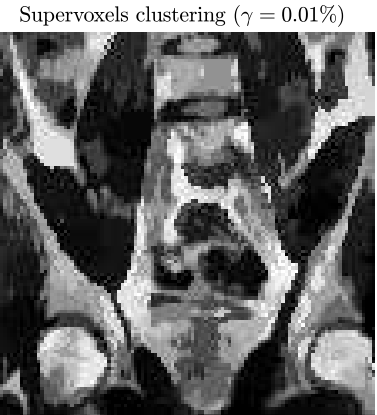

(d)

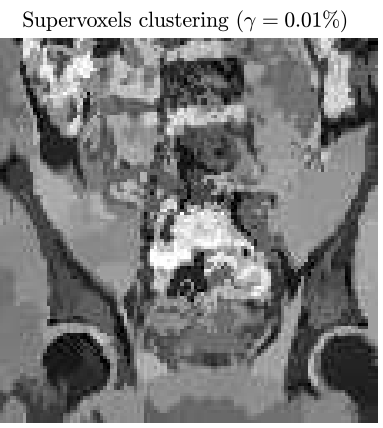

(h)

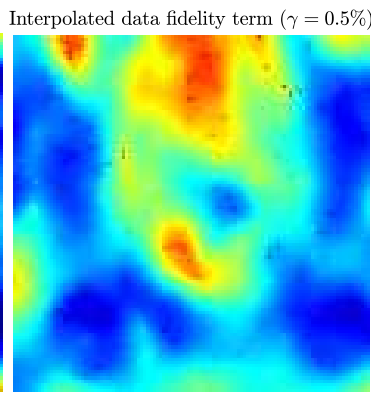

(j)

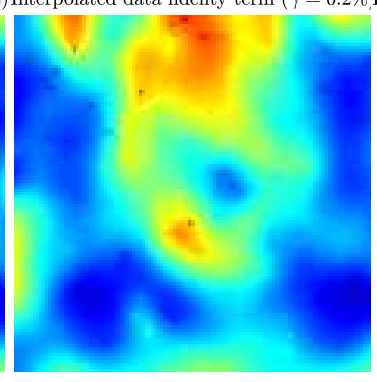

(k)

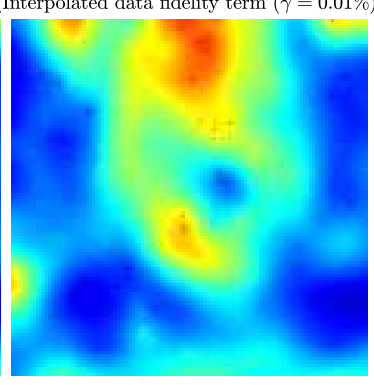

(1)

Figure 2: Typical data fidelity maps obtained with the abdominal T1/T2 MR-scans for various number of supervoxels. Images obtained with various voxel decimation ratios $\gamma=\{100 \%, 0.5 \%, 0.2 \%, 0.01 \%\}$ are displayed in the $1^{\text {st }}, 2^{\text {nd }}, 3^{\text {rd }}$ and $4^{\text {th }}$ columns, respectively (we recall that $\gamma$ is the ratio between the number of supervoxels and the amount of voxels in the original image). The first and the second rows show the clustered images $I$ (reference) and $J$ (image to register), respectively. For vizualisation, the image contrast was digitally enhanced, and grey color of each voxel in the displayed images was set to the mean grey color of the supervoxel region. The third row shows the corresponding interpolated data fidelity maps $D(T)$.

in (b-e) to clearly showcase the algorithm behavior for very low $\gamma$ values. Using DSEVo, a huge reduction of the computational latency was achievable ( $\simeq 89 \%$ ), however at the cost of a loss in terms of the average FEP ( $>15 \%)$. On the other hand, a similar registration accuracy together with a reduced computation time were obtained 


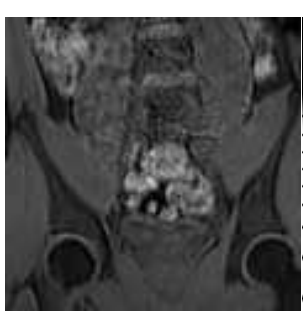

(a)

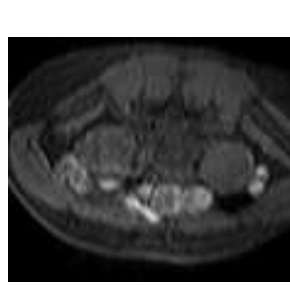

(f)

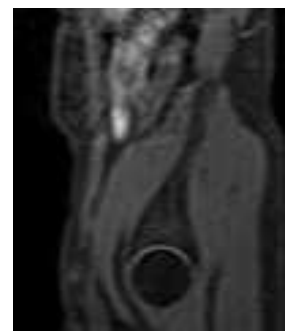

$(\mathrm{k})$

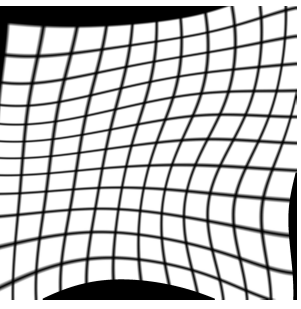

(b)
FEP (Before) $[\mathrm{mm}]$

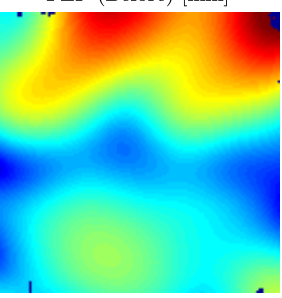

(c)

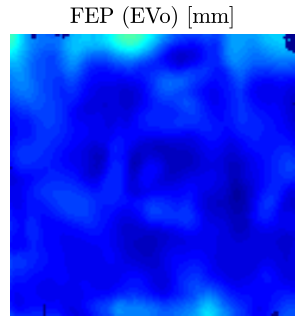

(d)

FEP $(\mathrm{EVo})[\mathrm{mm}]$

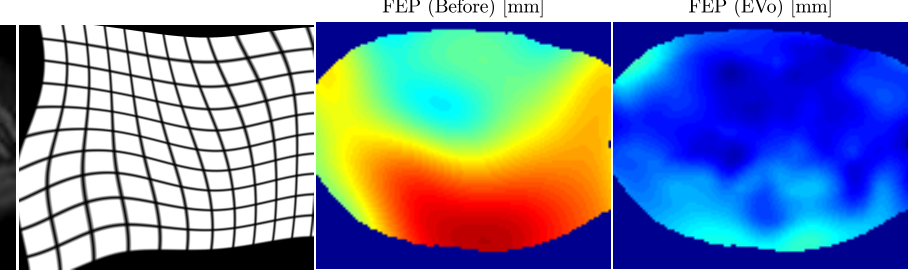

(g)

(h)

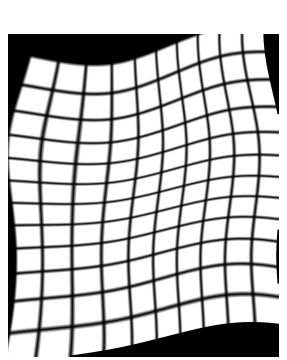

(1)
FEP (Before) [mm]

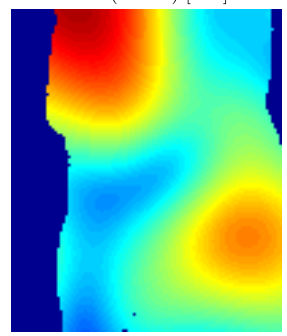

(m) (i)

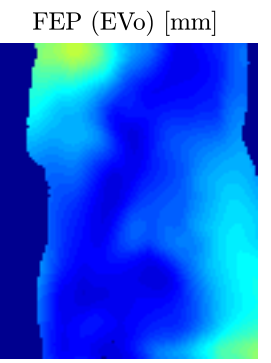

(n)
FEP (SV-EVo) $[\mathrm{mm}]$

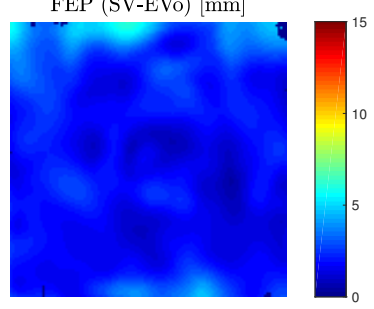

(e)

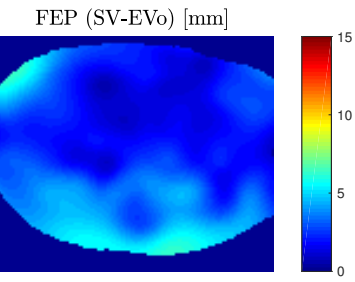

(j)

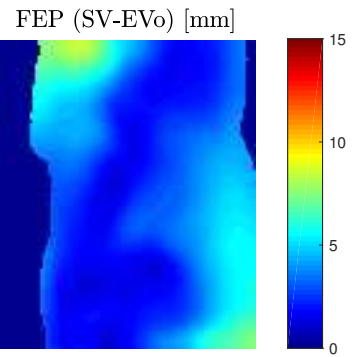

(o)

Figure 3: Example of registration results obtained using the abdominal T1/T2 MRscans. The $1^{\text {st }}$ column displays the image to register $(J)$. The $2^{\text {nd }}$ column displays the simulated deformation field (the displacement amplitudes were enlarged by a factor 3 for visualization). The voxel-wise FEP maps are displayed before registration ( $3^{\text {rd }}$ column), after registration using EVo ( $4^{\text {th }}$ column) and SV-Evo $\left(5^{\text {th }}\right.$ column, $\left.\gamma=1 \%\right)$. Coronal, transversal and sagittal images are displayed in the $1^{\text {st }}, 2^{\text {nd }}$ and $3^{\text {rd }}$ rows, respectively.

using SV-EVo for all tested voxel decimation ratio $\gamma$. In particular, the value $\gamma=1 \%$ provided a computational overhead decreased by $75 \%$ and $41 \%$ for the CPU and the GPU implementations, respectively.

\subsection{Annotated thorax inhale and exhale CT-scans (Study \#2)}

Fig. 5 shows an example of interpolated data fidelity terms obtained using the thorax CT-scans for various voxel decimation factors $\gamma$. The supervoxel clustering of a typical input image (Volunteer \#1) are reported in Fig. 2a-d (Task \#1). The visualization of the clusters was enhanced using the method described above (section 3.1). High image degradations are visible for $\gamma=0.01 \%$, especially around small anatomical structures such as the bronchus. The interpolated data fidelity maps $D(T)$, based on the values 


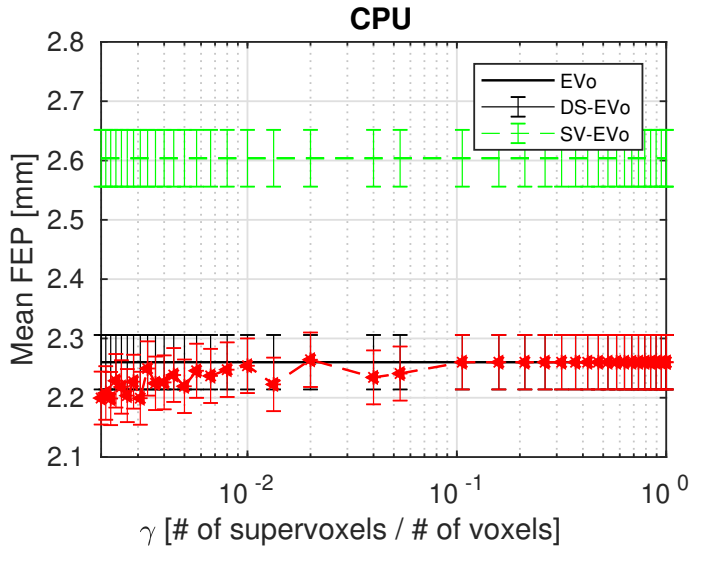

(a)

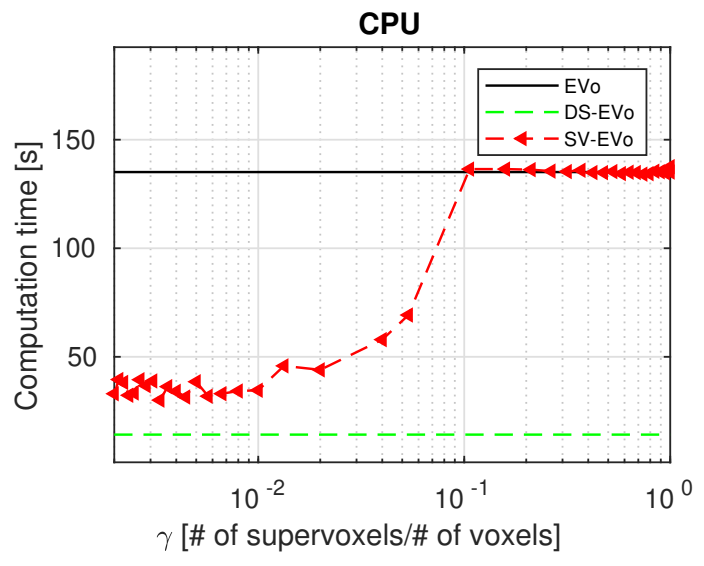

(c)

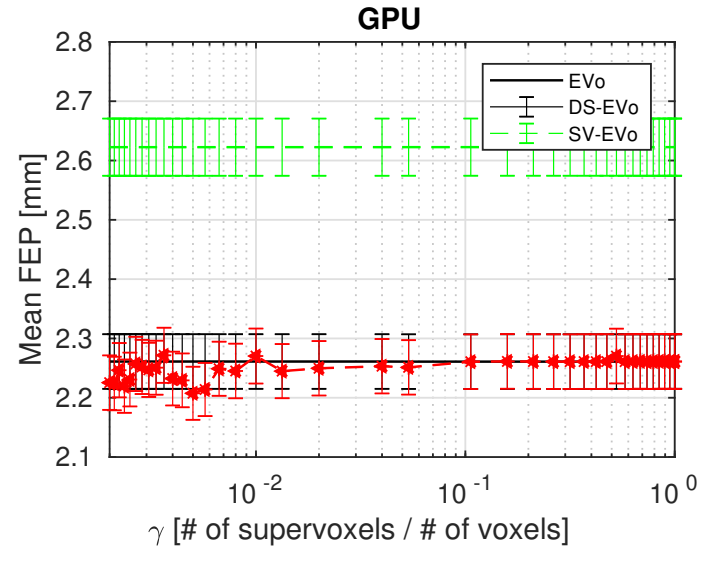

(b)

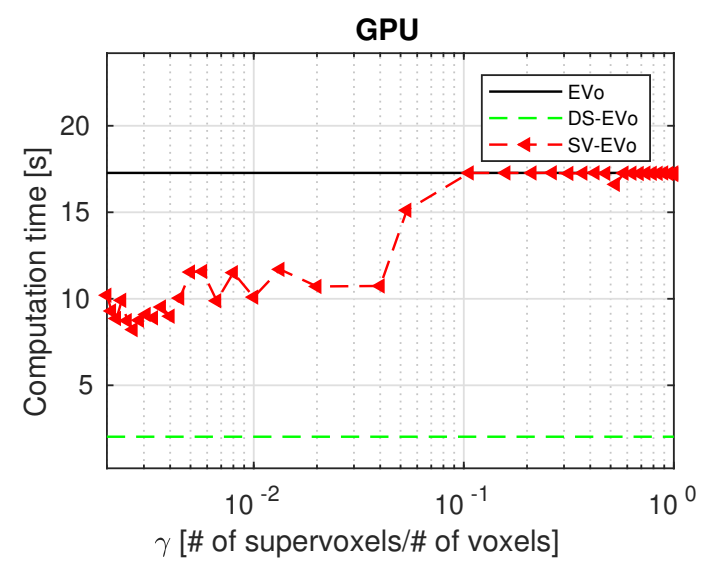

(d)

Figure 4: Evaluation of the impact of the voxel decimation on the performance of the proposed frame-work for the registration of the abdominal T1/T2 MR-scans undergoing an imposed deformation. The accuracy of the registration (a,b) and the reduction of the computational overhead (c,d) obtained using SV-EVo are reported for various values of $\gamma$. For each tested $\gamma$ value in (a) and (b), the standard error is displayed using vertical error bars. Values obtained using the CPU implementation are reported on the left (a,c) and values obtained using the GPU implementation are reported on the right (b,d). Results obtained using EVo (black solid line) and DS-EVo (green dashed line) are also reported for comparison.

computed on the subset of voxels (Task \#2 and \#3), are displayed in Fig. 2i-1. Although few differences in the image content in $D(T)$ are visible with $\gamma=0.2 \%, 0.5 \%$ (mainly high frequencies), large discrepancies are observable for $\gamma=0.01 \%$, especially around steep contours (such as in the lung/ribs/muscle frontiers).

Fig. 6a compares cumulative distributions of the TRE over 1500 landmarks (300 landmarks for 5 CT-scans) obtained before and after registration with EVo, SV-EVo $(\gamma=1 \%)$ and DS-EVo. All tested registration methods improve the landmark positions. It can be observed that similar error distributions are obtained over all landmarks using 


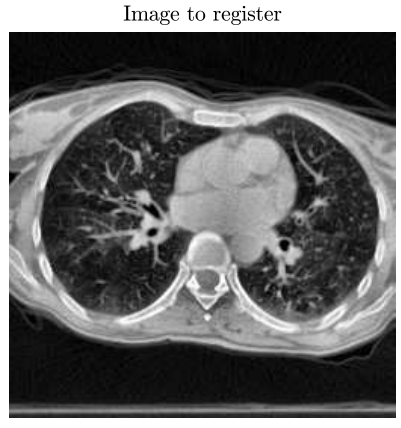

(a)

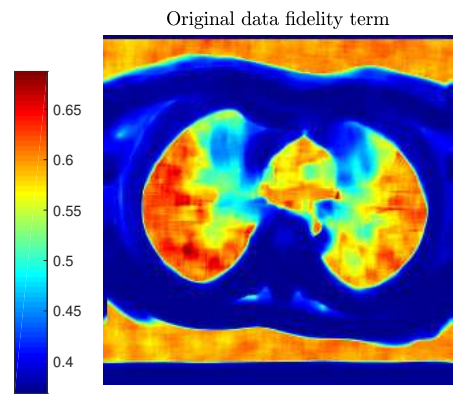

(f)

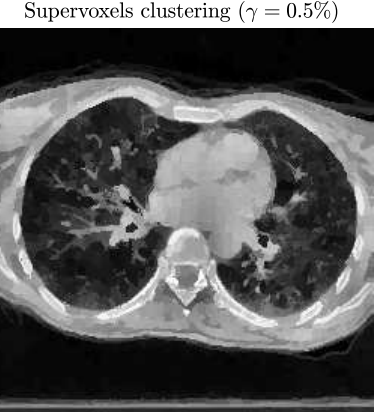

(c)

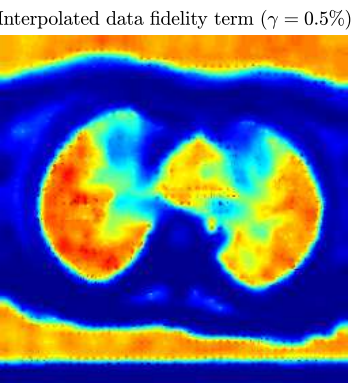

(h)

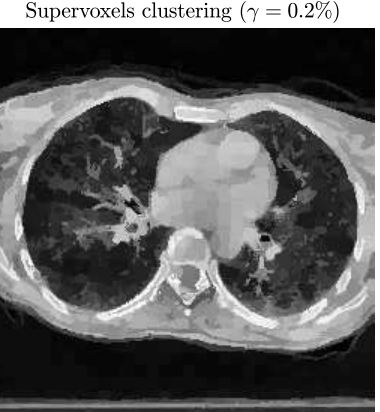

(d)
Supervoxels clustering $(\gamma=0.01 \%)$

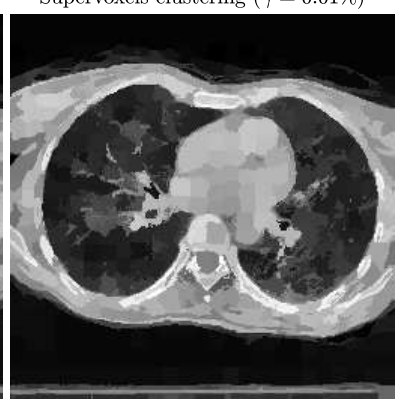

(e)

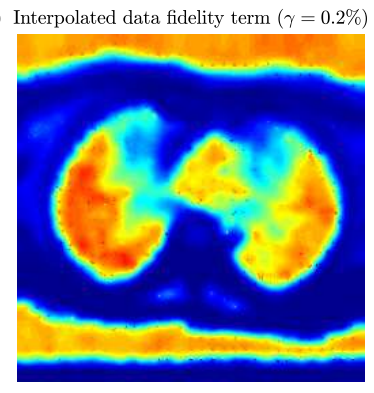

(i)

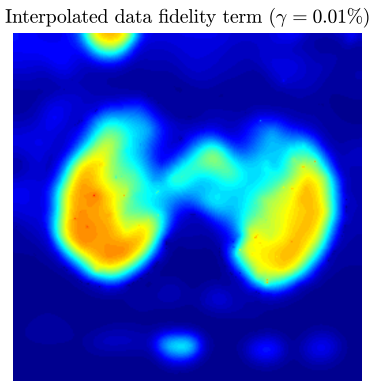

(j)

Figure 5: Typical data fidelity maps obtained with the thorax CT-scans for various number of supervoxels (Volunteer \#1). Images obtained with various voxel decimation ratios $\gamma=\{100 \%, 0.5 \%, 0.2 \%, 0.01 \%\}$ are displayed in the $1^{\text {st }}, 2^{\text {nd }}, 3^{\text {rd }}$ and $4^{\text {th }}$ columns, respectively. The first row shows an example of image clustering. For visualization, the image contrast was digitally enhanced, and the gray color of each voxel in the displayed images was set to the mean gray color of the supervoxel region. The second row shows the corresponding interpolated data fidelity maps $(D(T))$.

EVo and SV-EVo. A loss in motion estimate accuracy is however observable using DSEVo, as compared to EVo and SV-EVo. Fig. 6b-e analyze the performance of each tested algorithm in terms of accuracy of motion estimates and computation time. A log-scale is employed for the $\mathrm{X}$-axis in (b-e) to better illustrate the algorithm behavior for very low $\gamma$ values. In this experiment again, a considerable reduction of the computing cost was achievable using DS-EVo $(\simeq 88 \%)$, which comes however along with a notable loss in mean TRE (> 40\%). Using SV-EVo, a decrease of the computation time with minimal losses in terms of registration accuracy was obtained for $\gamma \geq 1 \%$. In particular, for $\gamma=1 \%$, the computational overhead decreased by $80 \%$ using the CPU implementation. A moderate speed-up $(\simeq 35 \%)$ was also obtained when using the GPU implementation. Below the $\gamma=1 \%$ threshold, it can be observed that the registration accuracy has a tendency to deteriorate with decreasing values of $\gamma$, as shown in Fig. 6b and 6c. 


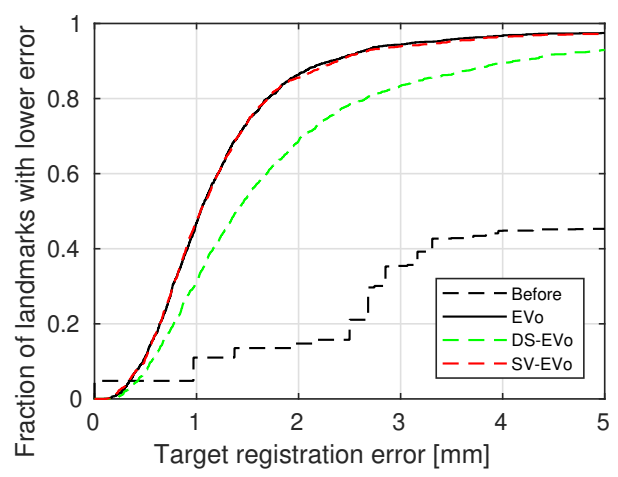

(a)

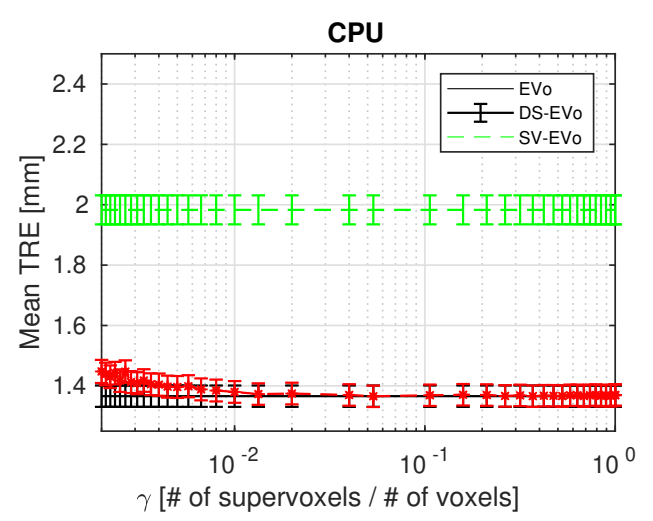

(b)

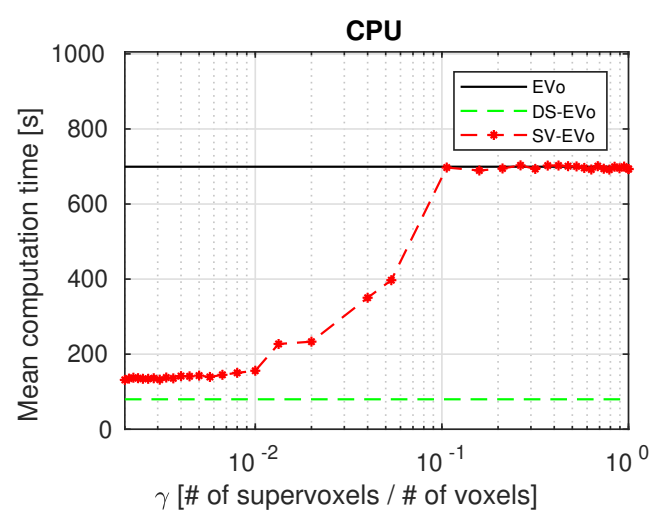

(d)

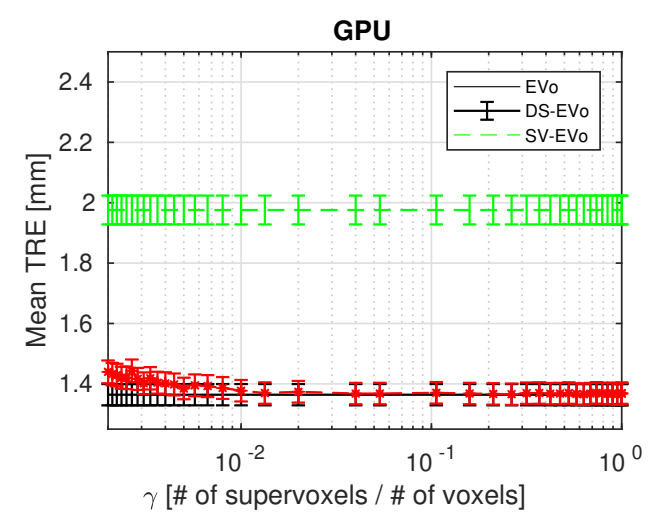

(c)

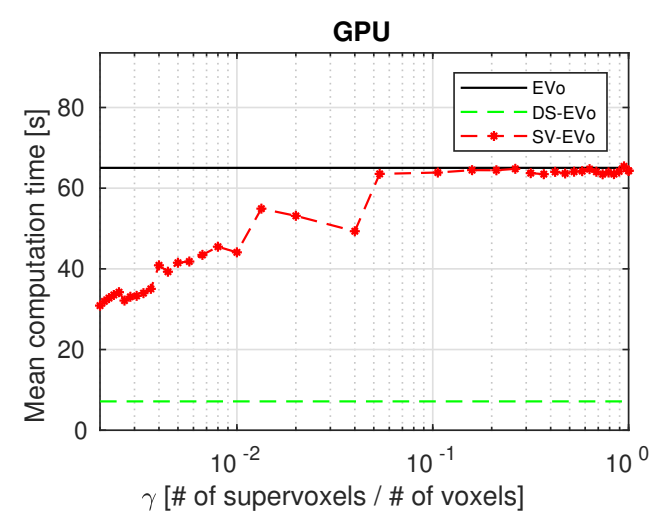

(e)

Figure 6: Registration of five cases of thorax CT-scans. (a): Typical cumulative distribution of target registration error obtained for a voxel decimation ratio $\gamma=1 \%$. (b-e): Evaluation of the impact of the voxel decimation on the performance of SV-EVo. The accuracy of the registration $(b, c)$ and the reduction of the computational overhead (d,e) obtained using SV-EVo are reported for various values of $\gamma$. For each tested $\gamma$ value in $(b, c)$, the standard error is displayed using vertical error bars. Values are reported using the CPU (b,d) and the GPU implementations (c,e). Results obtained using EVo (black solid line) and DS-EVo (green dashed line) are also reported for comparison. 
3.3. Correction of slow physiological drifts during MR-guided clinical interventions (Study \#3)

Fig. 7 illustrates the first step of the experimental protocol designed to analyze the benefit of using SV-EVo, as compared to EVo, for the estimation of slow physiological drifts in the abdomen: a gold standard is constructed using a mono-modal optical flow (OF) algorithm (Eq. (7)) applied on the T1-weighted data for both the reference and the image to register. A good correspondence can be observed between the kidneys and liver on the registered volumetric image of Fig. $7 \mathrm{~g}$-i and the region of interest manually segmented on the reference data. This indicates that the images have indeed been successfully registered. In addition, a spatially regular displacement, mainly leftright and moderately anterior-posterior, is observable in the inserts in Fig. 7d-f, which matches the visual observation of the organ motion in Fig. 7d-f.

A visualization of cross-constrast registration results can be seen in Fig. 8a-d. Fig. 8e-h summarizes, for all tested volunteers, the performance of the proposed method in terms of both accuracy of motion estimates and computational overhead. For each tested volunteer, both EVo and SV-EVo similarly reduce the mean FEP in both kidneys (8e) and liver (8f). However, the computational overhead with SV-EVo was reduced by $75 \%$ using the CPU (8g) and $31 \%$ using the GPU (8h), as compared to EVo.

\subsection{Paired abdominal CT/MR-scans (Study \#4)}

A visualization of a CT/T2-MRI registration result can be seen in Fig. 9. In the inserts, the CT image to register is shown in orange while the reference T2-MR image is shown in blue. In the registered case shown on the bottom, it can be observed that the liver and kidneys, which features a hyper-intense signal in the CT-scan (orange), matches closely the fat and the subcutaneous tissues, the latter being visible only in the T2-weighted image (blue). This indicates that the images have been successfully registered. This visual inspection is confirmed by the Fig. 10 which summarizes, for all five patients, the performance of the proposed method in terms of both accuracy of motion estimates and computational overhead. For each patient, both EVo and SV-EVo similarly increase the DSC of both body (10a) and kidneys (10b). However, the computational overhead with SV-EVo was reduced by $90 \%$ using the CPU (10c) and $70 \%$ using the GPU (10d), as compared to EVo.

\section{Discussion}

The proposed method addresses the issue of time consuming evaluations of the data fidelity term, which is typically required to perform multi-modal image crossregistration. The proposed idea is to swap, in a larger number of voxels, the calculation of the data fidelity term with a less computationally intensive regularized interpolation task. It must be underlined that our approach is only beneficial when two general conditions are met: First, the time cost arising from the regularized interpolation task 


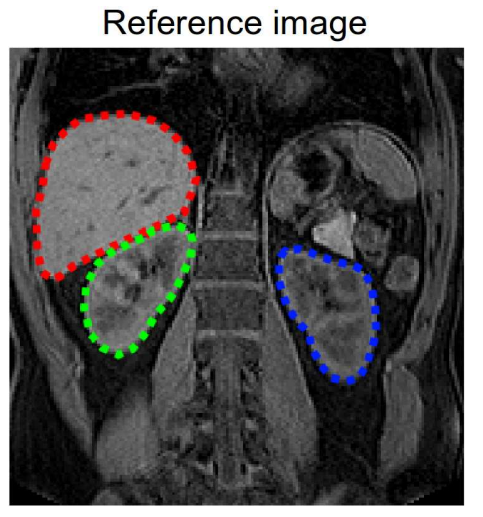

(a)

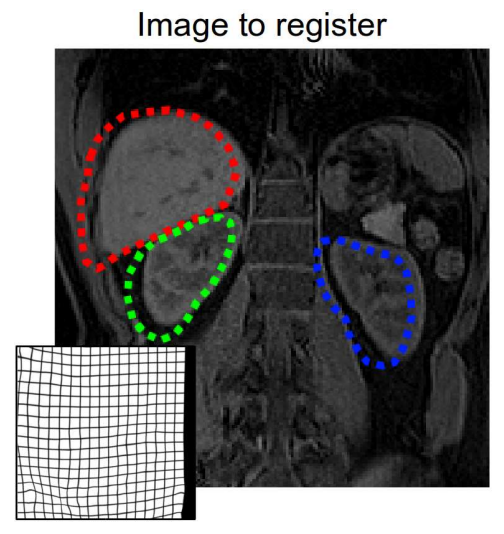

(d)

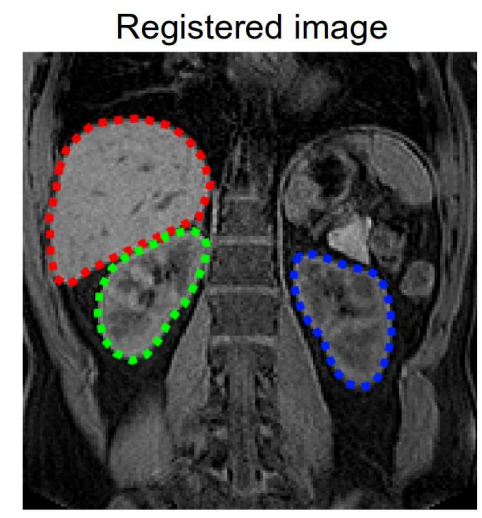

$(\mathrm{g})$

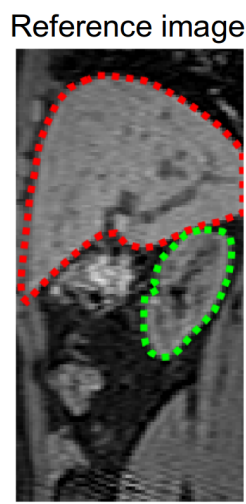

(b)

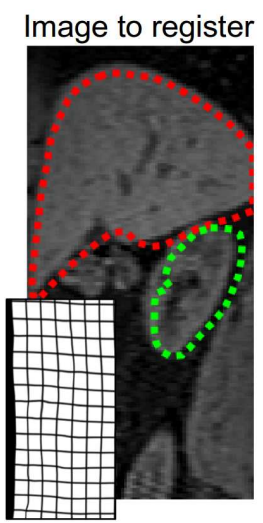

(e)

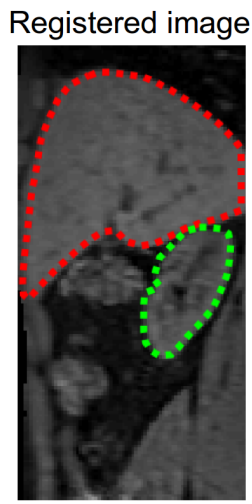

(h)

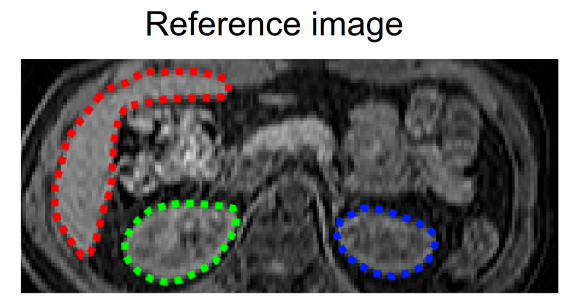

(c)

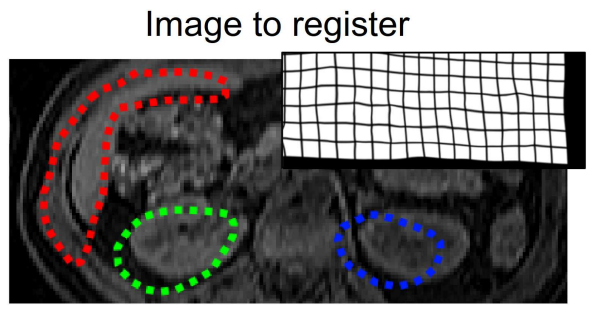

(f)

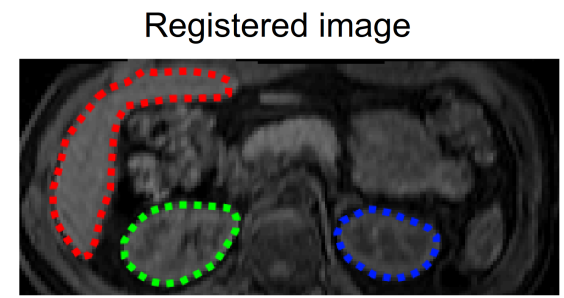

(i)

Figure 7: Example of a gold standard deformation, generated from the cross-contrast MR-data set showcasing slow physiological drifts (Volunteer \#4): an OF-based algorithm was applied on the T1-weighted data for both the reference and the image to register. Manually segmented ROI encompassing the right kidney, the left kidney and the liver are illustrated using green, blue and green dashed lines, respectively, in order to improve the visual inspection of the quality of the OF registration. The inserts in (d-f) show the estimated OF-deformation fields within the image FOV. 


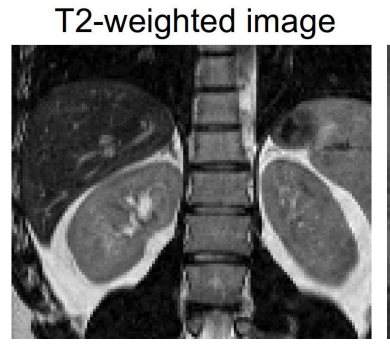

(a)

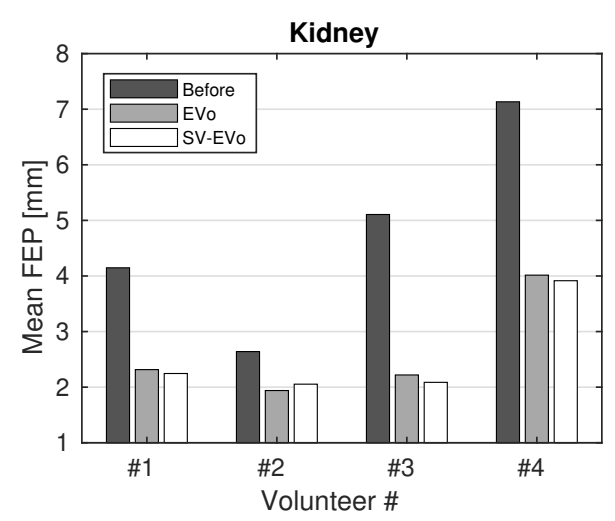

(e)

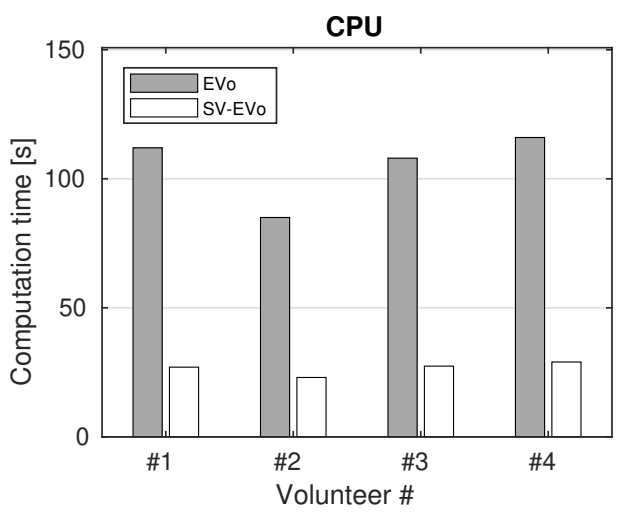

$(\mathrm{g})$

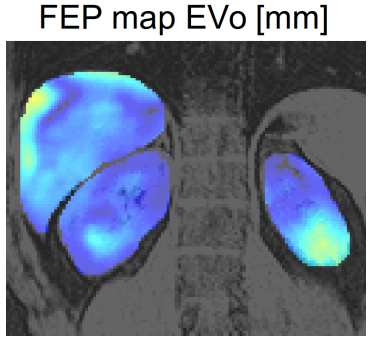

(c)

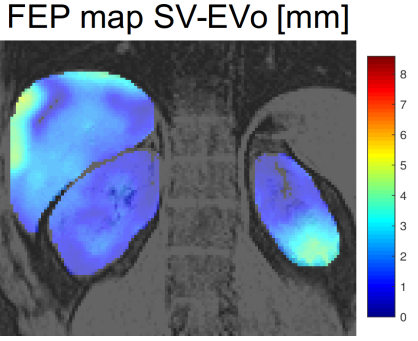

(d)

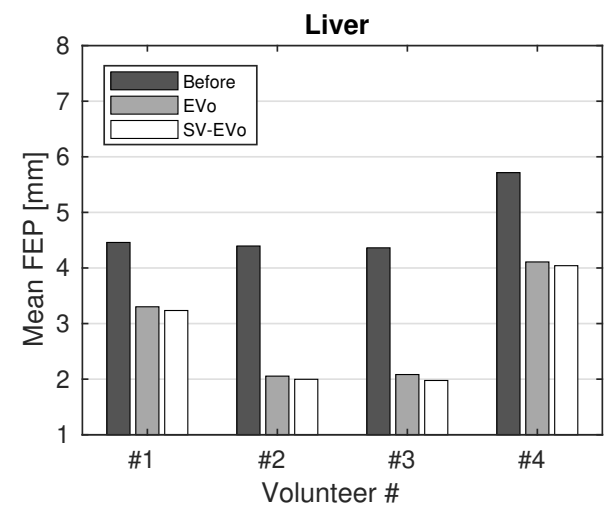

(f)

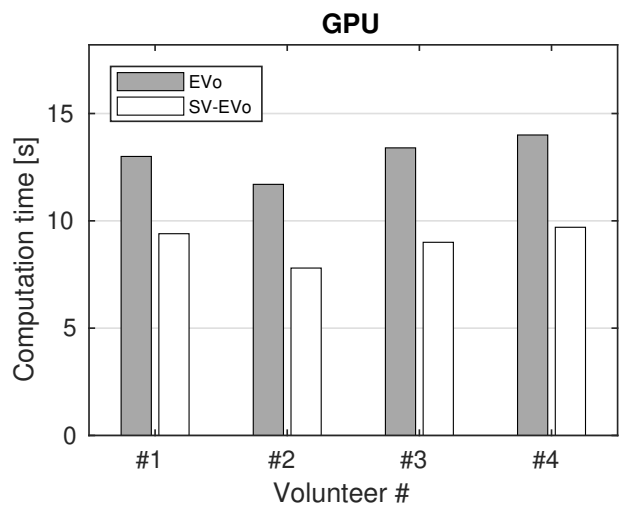

(h)

Figure 8: Benefit of the proposed method for the estimation of 3D slow physiological drifts on the four healthy volunteers. (a-d): Example of cross-contrast registration results obtained on the MR-data set with slow physiological drifts (Volunteer \#3). A coronal slice of the T2-weighted image to register is displayed in (a) before registration. The T1-weighted image, used as reference for the registration, is displayed in (b-d) with superimposed the voxel-wise FEP maps obtained before (b) and after registration using EVo (c) and SV-EVo (d). (e-h): The average registration errors within the two kidneys and the liver are reported for each volunteer in (e) and (f). The associated computation times are reported for the CPU (g) and the GPU (h) implementations. In these results, a typical voxel decimation ratio $\gamma$ equal to $1 \%$ was employed for SV-EVo. 


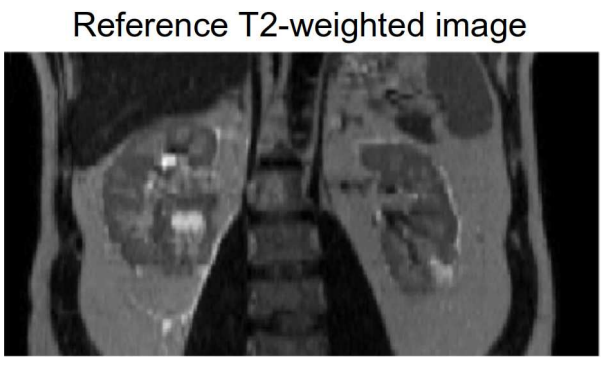

(a)

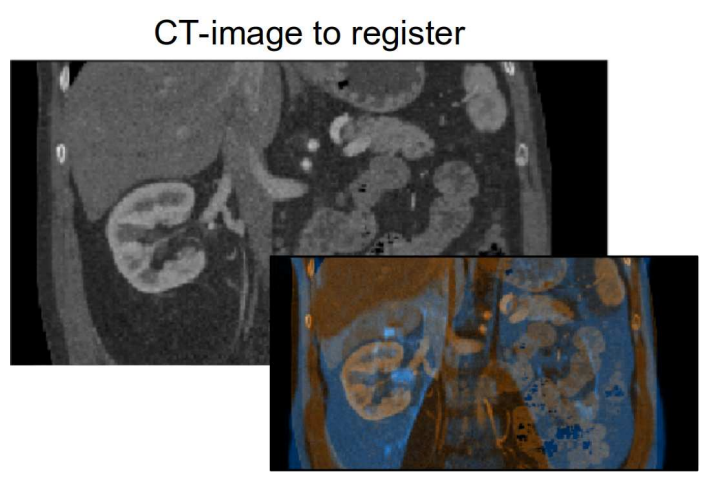

(c)

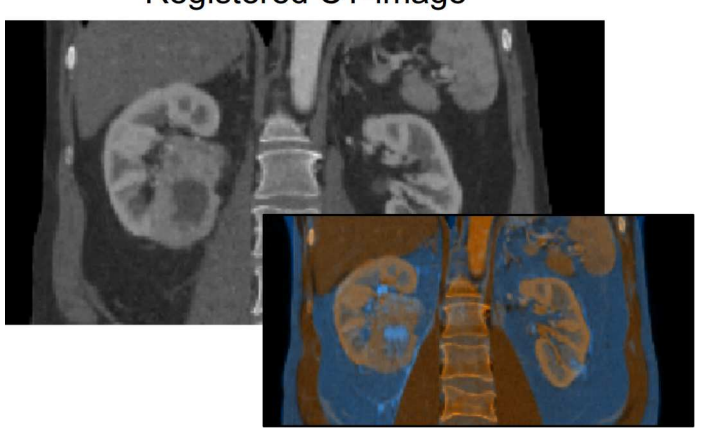

(e)

\section{Reference T2-weighted image}

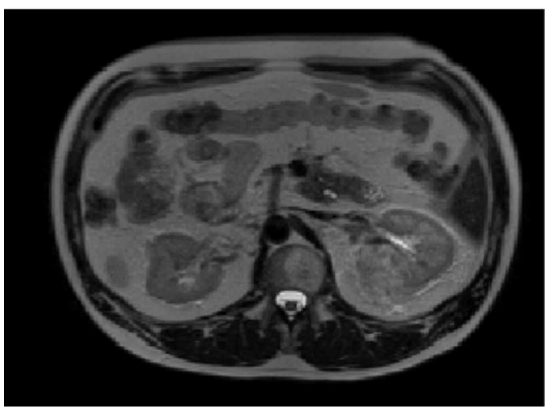

(b)

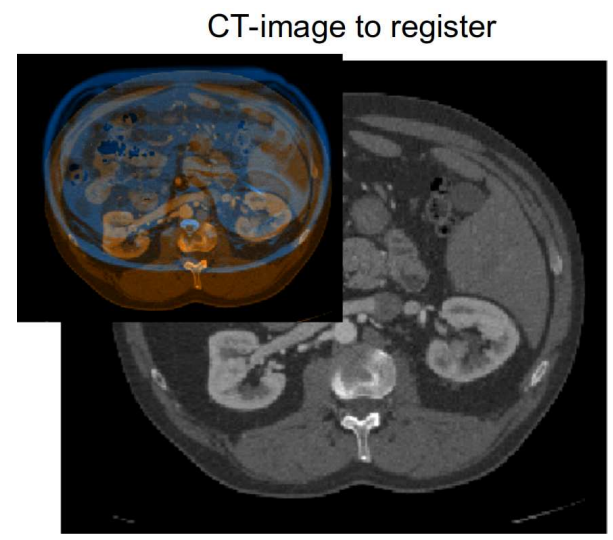

(d)

Registered CT-image

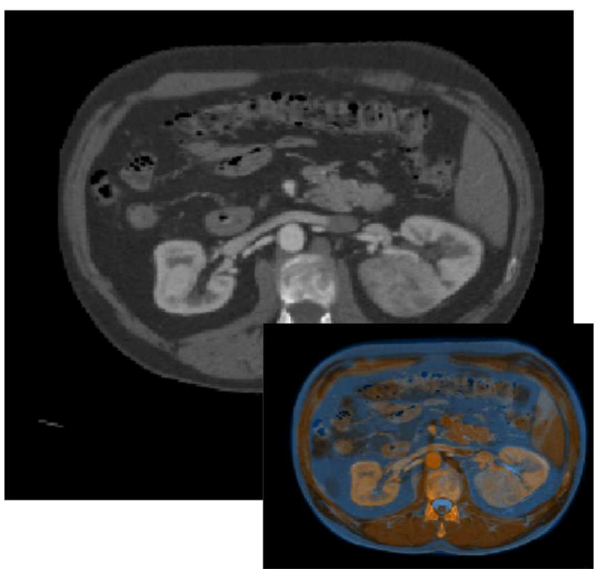

(f)

Figure 9: Example of multi-modal registration results obtained using SV-EVo on a pair of abdominal CT/T2-MRI data (Patient \#3). The T2-MRI, chosen as reference for the registration, is displayed in $(\mathrm{a}, \mathrm{b})$. The CT-image is reported before $(\mathrm{c}, \mathrm{d})$ and after $(\mathrm{e}, \mathrm{f})$ registration in coronal $(c, e)$ and transversal $(d, f)$ directions. In the inserts of $(c-f)$, the CT-image is displayed in orange and the T2-MRI in blue (complementary colour). In these results, a voxel decimation ratio $\gamma$ equal to $1 \%$ was employed. 


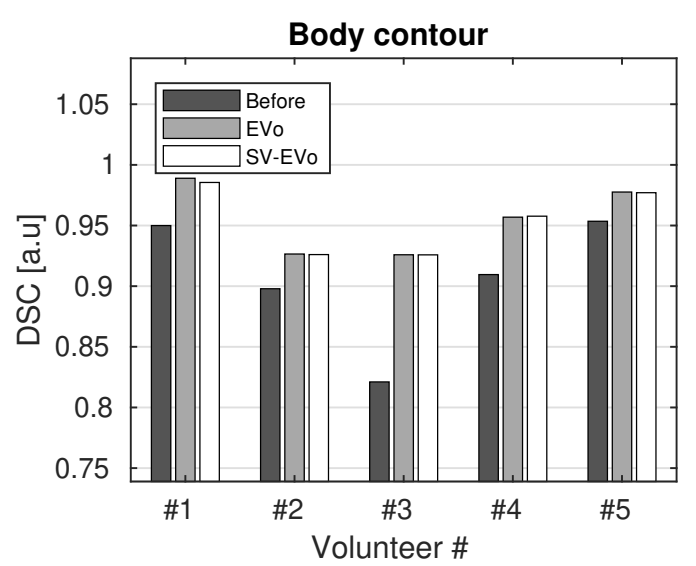

(a)

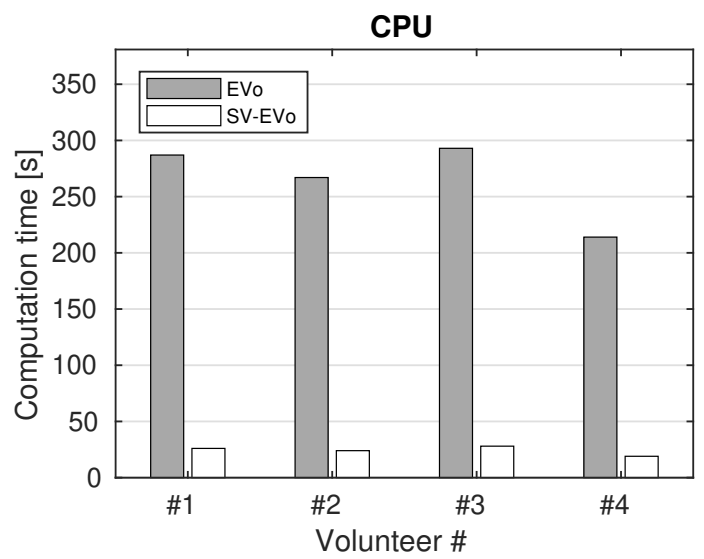

(c)

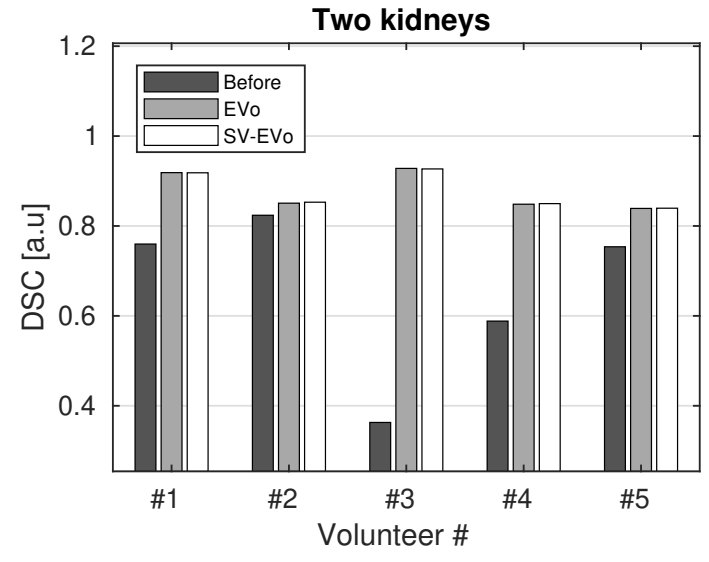

(b)

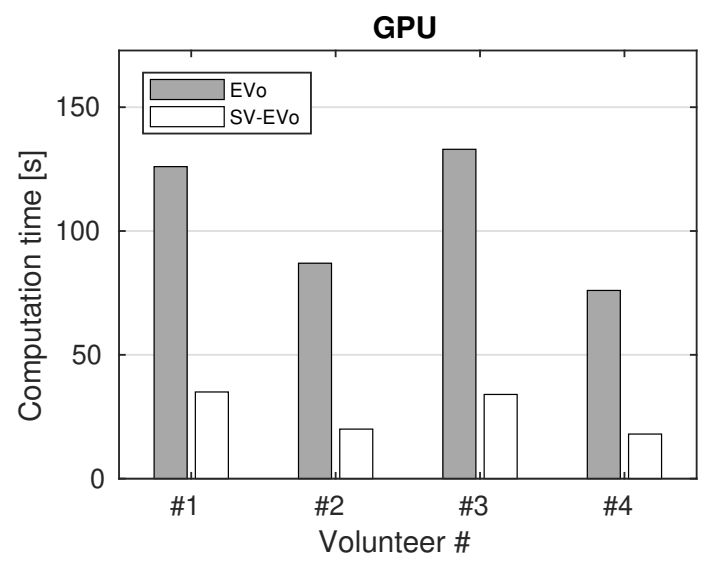

(d)

Figure 10: Benefit of the proposed method for the registration of multi-modal CT/T2MRI acquired on the five RCC patients. The DSC coefficients obtained using the body contour and the two kidneys are reported for each patient in (a) and (b). The associated computation times are reported for the CPU (c) and the GPU (d) implementations. In these results, a typical voxel decimation ratio $\gamma$ equal to $1 \%$ was employed for SV-EVo.

must be significantly lower than the one associated with the calculation of a data fidelity term. Second, the decimation of voxels involved in data fidelity calculations must not infer, at least to some extent, the amount of image content required for the registration process.

With regard to the first above-mentioned condition, the computation time gain, as a function of $\gamma$, depicts a steep slope in the interval $[0,10] \%$ (see Fig. 4c-d and 6d-e). This phenomenon was more pronounced using the CPU implementation (Fig. 4c and 6d) as compared to the GPU one (Fig. 4d and 6e). Beyond this point, the additional gain in computation time is minimal: any additional benefit of the voxel decimation is countered by the inherent growing number of iterations required in the regularized interpolation process to propagate $D_{s}(T)$ along the FOV.

Both above-mentioned conditions rely both on the amount and the exact spatial 
location of leading voxels selected in Tasks \#1 and \#2. In the scope of this study, we employed a supervoxel clustering algorithm which inherently increases the voxel spatial density in regions with larger amount of anatomical structures. Since the leading voxels are determined by the supervoxel algorithm, the only input parameter to tune is the amount of working voxels (defined by $\gamma$ in the scope of this paper).

The two above-mentioned conditions also rely on the accuracy of the regularized interpolation process in Task \#4. Interestingly, using the abdominal T1/T2 MR-scans, the removal of high frequencies in the data fidelity term prevents the optimization process to get caught into local minima, which has a small positive impact on the overall registration accuracy (see $4 \mathrm{a}$ and $4 \mathrm{~b}$ ). However, this observation does not apply to the thorax CT-scans for $\gamma<0.5 \%$ : large discrepancies in the data fidelity are observable when steep contours are present (see Fig. 5j), which hampers the overall registration accuracy (see Fig. 6b and 6c). It is noteworthy that the degradation of the accuracy is moderate, at least to some extent, since the algorithm is intrinsically designed to select leading voxels for the registration.

As a consequence, a compromise for $\gamma$ has to be found. At this point it is important to note that, although the impact of $\gamma$ on the registration accuracy and the computation time could be easily observed in studies \#1 and \#2 (see Fig. 4 and 6), this was unfortunately not the case for studies \#3 and \#4. The precision of such an analysis relies on the accuracy of the available gold standard. The latter was sufficient in study \#1 (the deformation was imposed and known) and study \#2 (a sufficient number of landmarks was available/three observers each). The precision of the gold standard was however hampered in study \#3 by the use of a third party optical flow algorithm, and in study \#4 by the limited knowledge of few anatomical tissue contours.

One can also note that DS-EVo, which corresponds to a uniform voxel decimation with a factor 2 along each spatial direction, would have an equivalent normalized decimation ratio of $1 / 8$ (i.e 12.5\%). However, using $\gamma=12.5 \%$, SV-EVo provides accuracy and computation time similar to EVo, as shown in Fig. 4 and 6: the decimation is here not sufficient to compensate for additional costs arising from the regularized interpolation task.

On the presented experiments, using the proposed implementation, the tradeoff on $\gamma$ was typically found for $\gamma=1 \%$. In this case, for the tested data sets and using the CPU implementation, SV-EVo provided motion estimates with an accuracy similar to EVo together with computational overhead close to DS-EVo (see Fig. 4c, 6d, 8g and 10c). Concerning the GPU implementation, the benefit of the proposed method was more moderate. This is due to the fact that the highly parallel and distributed architecture of GPU allows reducing intrinsically the contribution of the time cost associated to the data fidelity term. Moreover, additional fixed time costs associated to data transfer from the host to the GPU device memory further hampered the overall benefit of the proposed data fidelity term optimization. A great speed-up around 30-40 \% was however still achievable for $\gamma=1 \%$, as shown in Fig. $4 \mathrm{~d}, 6 \mathrm{e}, 8 \mathrm{~h}$ and $10 \mathrm{~d}$.

It must be however underlined that there is no easy approach to establish the 
correct value of the decimation $\gamma$ without having any sort of silver or gold standard. The authors believe that the use of quality assurance criteria (QA) on the estimated motion, as proposed in (Zachiu et al. 2018), is a promising path of investigation.

\section{Conclusion}

A complete variational framework was introduced to improve the computational performance of the recently proposed multi-modal image registration algorithm EVolution (Denis de Senneville et al. 2016). The proposed approach prioritizes voxels of interest for the registration process, based on a supervoxel clustering algorithm. Costly calculations are hereby restrained to a subset of voxels, leading to a less expensive spatial regularized interpolation process. Using a voxel decimation ratio of $\gamma=1 \%$, the required computation time on the presented data sets were reduced by $75 \%$ and $30 \%$, using the CPU and the GPU implementations respectively, with no negative impact on the accuracy. In particular, approximately $25 \mathrm{~s}$ and $9 \mathrm{~s}$ were necessary for the registration of a pair of $192 \times 192 \times 75 \mathrm{~T} 1 / \mathrm{T} 2-\mathrm{MR}$ volumes using the employed CPU and GPU hardwares, respectively (against $120 \mathrm{~s}$ and $13 \mathrm{~s}$ for EVolution).

It must be emphasized that inherent limitations of the original EVolution algorithm still apply here: in particular, the impact of shearing motions may not be negligible (ex: sliding of liver and kidneys on the abdominal walls), and will need to be addressed in future works. We also expect that the proposed framework can be adapted to other image registration algorithms in order to reduce computationally intensive calculations.

\section{Acknowledgment}

Experiments presented in this paper were carried out using the PlaFRIM experimental testbed, supported by Inria, CNRS (LABRI and IMB), Université de Bordeaux, Bordeaux INP and Conseil Régional d'Aquitaine (see https://www.plafrim.fr/). The authors thank the Laboratory of Excellence TRAIL ANR-10-LABX-57 for funding. This study has been carried out with the financial support of the French National Research Agency (ANR) in the frame of the "Investments for the future" Programme IdEx Bordeaux-CPU (ANR-10-IDEX-03-02). This work was also supported in part by ITEA 3, project no. 16016 (STARLIT).

\section{Appendix A. Regularized interpolation of the sparse data fidelity term $D_{s}(T)($ Task \#4)}

This appendix details the numerical scheme employed in Task \#4 (section 2.1.4) to calculate a spatial regular data fidelity $D(T)$ from the sparse data fidelity term $D_{s}(T)$ using Eq. (5).

In order to render Eq. (5) differentiable, one can replace $|s|$ by $\psi(s)=\sqrt{s^{2}+\epsilon^{2}}$, with $\epsilon$ a small positive constant and $s=D(T)-D_{s}(T)$. The Euler-Lagrange equations 
applied on $E(D(T))$ provide the following equation for each $\vec{r} \in \Omega$ :

$$
\frac{\xi D(T)-D_{s}(T)}{\psi\left(\xi D(T)-D_{s}(T)\right)}-\mu \Delta D(T)=0
$$

where $\Delta$ denotes the Laplacian operator (Gonzalez \& Woods 2006). As suggested in (Horn \& Schunck 1981), $\Delta($.$) can be approximated in the discrete domain by \Delta f=\bar{f}-f$, $\bar{f}$ being the $3 \times 3 \times 3$ local average of a continuous and differentiable function $f . D(T)$ can thus be found iteratively for each $\vec{r} \in \Omega$ through the following explicit fixed-point scheme:

$$
D(T)^{n+1}=\overline{D(T)}^{n}-\mu^{-1} \frac{\xi D(T)^{n}-D_{s}(T)}{\psi\left(\xi D(T)^{n}-D_{s}(T)\right)}
$$

where $n+1$ denotes the new iteration. Practically, Eq. (A.2) can be approximated as follows:

$$
D(T)^{n+1}=\left\{\begin{array}{cl}
\overline{D(T)}^{n} & \text { if } \xi=0 \\
\overline{D(T)}^{n}-\mu^{-1} \operatorname{sign}\left(D(T)^{n}-D_{s}(T)\right) & \text { if } \xi=1
\end{array}\right.
$$

At each iteration, $\mu$ was adjusted to enforce $D(T)^{n+1}$ to match non-zero values in $D_{s}(T)$. That way, $D(T)$ could thus be automatically determinated iteratively as follows:

$$
D(T)^{n+1}= \begin{cases}\overline{D(T)}^{n} & \text { if } \xi=0 \\ D_{s}(T) & \text { if } \xi=1\end{cases}
$$

The residual $\left\|D(T)^{n}-D(T)^{n-1}\right\|_{2}$ was compared to a maximal allowed tolerance of $10^{-3}$ to ensure the convergence.

\section{References}

Achanta, R., Shaji, A., Smith, K., Lucchi, A., Fua, P. \& Ssstrunk, S. (2012). Slic superpixels compared to state-of-the-art superpixel methods, IEEE Transactions on Pattern Analysis and Machine Intelligence 34(11): 2274-2282.

Baker, S., Scharstein, D., Lewis, J. P., Roth, S., Black, M. J. \& Szeliski, R. (2011). A database and evaluation methodology for optical flow, International Journal of Computer Vision 92(1): 1-31.

Brock, K. K., Mutic, S., McNutt, T. R., Li, H. \& Kessler, M. L. (2017). Use of Image Registration and Fusion Algorithms and Techniques in Radiotherapy: Report of the AAPM Radiation Therapy Committee Task Group No. 132, Med Phys 44(7): e43 - e76.

Castillo, E., Castillo, R., Guerra, R., Johnson, V., McPhail, T., Garg, A. \& Guerrero, T. (2009). A framework for evaluation of deformable image registration spatial accuracy using large landmark point sets, Physics in Medicine and Biology 54: 1849-1870.

Castillo, E., Castillo, R., Martinez, J. \& Guerrero, T. (2010b). Ventilation from four-dimensional computed tomography: density versus jacobian methods, Physics in Medicine and Biology $55(16)$.

Castillo, E., Castillo, R., Martinez, J., Shenoy, M. \& Guerrero, T. (2010a). Four-dimensional deformable image registration using trajectory modeling, Physics in Medicine and Biology 55: $305-327$. 
Degen, J. \& Heinrich, M. P. (2016). Multi-atlas based pseudo-ct synthesis using multimodal image registration and local atlas fusion strategies, 2016 IEEE Conference on Computer Vision and Pattern Recognition Workshops (CVPRW), pp. 600-608.

Denis de Senneville, B., Zachiu, C., Ries, M. \& Moonen, C. T. W. (2016). Evolution: an edgebased variational method for non-rigid multi-modal image registration, Physics in Medicine and Biology 61(20): 7377.

Dice, L. R. (1945). Measures of the amount of ecologic association between species, Ecology 26(3): 297302.

Donné, S., Aelterman, J., Goossens, B. \& Philips, W. (2015). Fast and robust variational optical flow for high-resolution images using slic superpixels, Advanced Concepts for Intelligent Vision Systems, Springer International Publishing, pp. 205-216.

Gkamas, T. \& Nikou, C. (2011). Guiding optical flow estimation using superpixels, 2011 17th International Conference on Digital Signal Processing (DSP), pp. 1-6.

Glitzner, M., Denis de Senneville, B., Lagendijk, J. J. W., Raaymakers, B. W. \& Crijns, S. P. M. (2015). On-line 3 D motion estimation using low resolution MRI, Physics in Medicine and Biology 60(16): N301.

Gonzalez, R. \& Woods, R. (2006). Digital Image Processing (3rd Edition), Prentice-Hall, Inc.

Guckenberger, M., Richter, A., Boda-Heggemann, J. \& Lohr, F. (2012). Motion compensation in radiotherapy, Critical Reviews and trade; in Biomedical Engineering 40(3): 187-197.

Haber, E. \& Modersitzki, J. (2006). Intensity gradient based registration and fusion of multi-modal images, in R. Larsen, M. Nielsen \& J. Sporring (eds), Medical Image Computing and ComputerAssisted Intervention - MICCAI 2006, Springer Berlin Heidelberg, Berlin, Heidelberg, pp. 726733.

Haddad, A. \& Meyer, Y. (2004). Variational methods in image processing, Technical Report, UCLA CAM Reports .

Heinrich, M., Jenkinson, M., Bhushan, M., Matin, T., Gleeson, F., Brady, S. \& Schnabel, J. (2012). MIND: Modality independent neighbourhood descriptor for multi-modal deformable registration, Medical Image Analysis 16(7): 1423-1435.

Hodneland, E., Lundervold, A., Rorvik, J. \& Munte-Kaas, A. Z. (2014). Normalized gradient fields for nonlinear motion correction of DCE-MRI time series, Comput Med Imaging Graph 38(3): 202 210.

Horn, B. \& Schunck, B. (1981). Determining optical flow, Artificial intelligence 17: 185-203.

Jolesz, F. A. \& Hynynen, K. (2002). Magnetic resonance image-guided focused ultrasound surgery, Cancer J 8(1): 100-12.

Klein, S., van der Heide, U. A., Lips, I. M., van Vulpen, M., Staring, M. \& Pluim, J. P. (2008). Automatic segmentation of the prostate in 3D MR images by atlas matching using localized mutual information, Med Phys 35(4): 1407 - 1417.

König, L., Kipshagen, T. \& Rühaak, J. (2014). A non-linear image registration scheme for real-time liver ultrasound tracking using normalized gradient fields, MICCAI Challenge on Liver Ultrasound tracking, Boston, USA.

Loeckx, D., Slagmolen, P., Maes, F., Vandermeulen, D. \& Suetens, P. (2010). Nonrigid image registration using conditional mutual information, IEEE Trans Med Imaging 29(1): 19 - 29.

Maes, F., Collignon, A., Vandermeulen, D., Marchal, G. \& Suetens, P. (1997). Multimodality image registration by maximization of mutual information, IEEE Transactions on Medical Imaging 16(2): 187-198.

Maurer, C., Fitzpatrick, J., Wang, M., Galloway, R., Maciunas, R. \& Allen, G. (1997). Registration of head volume images using implantable fiducial markers, IEEE Transactions on Medical Imaging 16: $447-462$.

NVIDIA (2008). NVIDIA CUDA: compute unified device architecture, Programming guide, NVIDIA Corporation, 2.0 edition .

Pluim, J., Maintz, J. \& Viergever, M. (2003). Mutual-information-based registration of medical images: 
A survey, IEEE Transactions on Medical Imaging 22(8): 986-1004.

Ramani, S., Thevenaz, P. \& Unser, M. (2010). Regularized interpolation for noisy images, IEEE Transactions on Medical Imaging 29(2): 543-558.

Rivaz, H., Karimaghaloo, Z. \& Collins, D. L. (2014). Self - similarity weighted mutual information: A new nonrigid image similarity metric, Med Image Anal 18(2): 343 - 358.

Rohlfing, T., Maurer, C. R. J., Bluemke, D. A. \& Jacobs, M. A. (2003). Volume - Preserving Nonrigid Registration of MR Breast Imaged Using Free - Form Deformation with an Incompressibility Constraint, IEEE Trans Med Imaging 22(6): 730 - 741.

Rubeaux, M., Simon, A., Gnep, K., Colliaux, J., Acosta, O., de Crevoisier, R. \& Haigron, P. (2013). Evaluation of non-rigid constrained CT/CBCT registration algorithms for delineation propagation in the context of prostate cancer radiotherapy, Medical Imaging 2013: Image-guided procedures, robotic interventions and modeling, Vol. 8671, SPIE Proceedings.

Studholme, C., Drapaca, C., Iordanova, B. \& Cardenas, V. (2006). Deformation-based mapping of volume change from serial brain MRI in the presence of local tissue contrast change, IEEE Trans Med Imaging 25(5): 626 - 639 .

Studholme, C., Hill, D. L. G. \& Hawkes, D. J. (1999). An overal invariant entropy measure of 3D medical image alignment, Pattern Recognition 32(1): 71 - 86.

Veksler, O., Boykov, Y. \& Mehrani, P. (2010). Superpixels and supervoxels in an energy optimization framework, Proceedings of the 11th European Conference on Computer Vision: Part V, ECCV'10, Springer-Verlag, Berlin, Heidelberg, pp. 211-224.

Viola, P. \& Wells III, W. (1997). Alignment by maximization of mutual information, International Journal of Computer Vision 24: 137-154.

Weickert, J., Bruhn, A., Papenberg, N. \& Brox, T. (2003). Variational optic flow computation: From continuous models to algorithms, International Workshop on Computer Vision and Image Analysis (ed. L. Alvarez), IWCVIA03, Las Palmas de Gran Canaria.

Zachiu, C., Denis de Senneville, B., Dmitriev, I. D., Moonen, C. T. W. \& Ries, M. (2017a). A framework for continuous target tracking during MR-guided high intensity focused ultrasound thermal ablations in the abdomen, Journal of Therapeutic Ultrasound 5(1): 27.

Zachiu, C., Denis de Senneville, B., Moonen, C. T. W., Raaymakers, B. W. \& Ries, M. (2018). Anatomically plausible models and quality assurance criteria for online mono- and multi-modal medical image registration, Physics in Medicine and Biology 63(15): 155016.

Zachiu, C., Denis de Senneville, B., Ries, M. \& Moonen, C. T. W. (2015). A framework for the correction of slow physiological drifts during MR-guided HIFU therapies: Proof of concept, Medical Physics 42(7): 4137-4148.

Zachiu, C., Denis de Senneville, B., Tijssen, R. H. N., Kotte, A. N. T. J., C., H. A., Kerkmeijer, L. G. W., Lagendijk, J. J. W., Moonen, C. T. W. \& Ries, M. G. (2017b). Non-rigid CT/CBCT to $\mathrm{CBCT}$ registration for online external beam radiotherapy guidance, Physics in Medicine and Biology .

Zachiu, C., Papadakis, N., Ries, M., Moonen, C. T. W. \& Denis de Senneville, B. (2015). An improved optical flow tracking technique for real-time MR-guided beam therapies in moving organs, Physics in Medicine and Biology 60(23): 9003.

Zachiu, C., Ries, M., Ramaekers, P., Guey, J. L., Moonen, C. T. W. \& Denis de Senneville, B. (2017c). Real-time non-rigid target tracking for ultrasound-guided clinical interventions, Physics in Medicine and Biology 62(20): 8154.

Zitová, B. \& Flusser, J. (2003). Image Registration Methods: A Survey, Image and Vision Computing 21(11): $977-1000$.

Zuiderveld, K. (1994). Contrast limited adaptive histogram equalization, in P. S. Heckbert (ed.), Graphics Gems IV, Academic Press Professional, Inc., San Diego, CA, USA, pp. 474-485. 